\title{
Flows Induced by Coriolis-Influenced Vertically Propagating Two-Dimensional Internal Gravity Wavepackets
}

\author{
Bruce R. Sutherland, ${ }^{1,2, *}$ Wyatt Reeves, ${ }^{1}$ and Ton S. van den Bremer ${ }^{3}$ \\ ${ }^{1}$ Department of Physics, University of Alberta, \\ Edmonton, Alberta T6G 2E1, Canada \\ ${ }^{2}$ Department of Earth \& Atmospheric Sciences, \\ University of Alberta, Edmonton, Alberta T6G 2E3, Canada \\ ${ }^{3}$ Department of Engineering Science, \\ University of Oxford, Oxford OX1 3PJ, UK
}

(Dated: June 10, 2020)

\begin{abstract}
Theory is developed to predict the flows induced by small-amplitude two-dimensional vertically propagating internal wavepackets under the influence of rotation. While the long wave response originally predicted by Bretherton [J. Fluid Mech. 36, 785-803 (1969)] dominates if the influence of background rotation is negligible, the induced waves are found to be evanescent if the Coriolis parameter is sufficiently large. Explicitly, evanescent induced flows dominate if the Coriolis parameter is greater than the forcing frequency, which is set by the ratio of the vertical group velocity of the wavepacket divided by the vertical scale of modulation of the wavepacket. The predicted structure of the induced flow is confirmed by numerical simulations. For an up- and rightward propagating Gaussian wavepacket that dominantly induces a long wave response, the flow is rightward across the upper flank and leftward across the lower flank of the wavepacket. In contrast, the induced flow for a dominantly evanescent response is negative over the middle of the wavepacket. This qualitative change in structure is anticipated to influence the modulational stability of moderately large amplitude wavepackets.
\end{abstract}




\section{INTRODUCTION}

Internal gravity waves propagate due to buoyancy forces within a stratified fluid. One class of internal gravity waves is confined to an interface or bounded above and below such that, in either case, the waves propagate only in the horizontal. The second class of internal gravity waves propagate vertically as well as horizontally in continuously stratified fluid. Our interest here lies in the latter class of waves, which we hereafter refer to simply as "internal waves". Although there is no Stokes drift associated with these waves, it is well known that they induce an Eulerian flow ${ }^{1}$ whose qualitative structure depends upon the relative aspect ratios of the wavepacket ${ }^{2}$. In particular, Bretherton ${ }^{3}$ first showed that a two-dimensional (spanwise-infinite) wavepacket induces an Eulerian flow in the form of long internal waves that trail the wavepacket to its left and right. Likewise, a three-dimensional wavepacket induces a long wave response if it is sufficiently wide in the spanwise direction ${ }^{2,4}$. For internal waves influenced by the Earth's rotation, it is well known that their frequency is restricted to lie between the Coriolis frequency, $f$ and the background buoyancy frequency, $N$. Extending previous work examining Eulerian flows induced by two-dimensional wavepackets unaffected by background rotation ${ }^{3,5}$, here we include the influence of the Coriolis force, anticipating that the long wave response should become evanescent if this force is sufficiently strong.

In part due to the need to understand pathways for energy transport from large-scale atmospheric and oceanic circulations to small dissipative scales, there have been several studies aiming to examine interactions between near-inertial waves (internal waves with frequency close to $f$ ) and mean flows ${ }^{6-11}$. In most of these studies, the focus has been on the excitation of inertial waves by geostrophically balanced flows. Conversely, even relatively large frequency internal waves can put energy into large scale flows due to dissipative processes, such as overturning and breaking ${ }^{12-16}$, as well as non-dissipative processes, such as Eulerian induced flows ${ }^{6,10,17-19}$. It is an investigation of the latter case that guides the work presented herein.

Notably, Tabaei \& Akylas $^{4}$ performed a comprehensive examination of flows induced by two- and three-dimensional internal wavepackets including the influence of rotation and weakly nonlinear effects associated with moderately large amplitude waves. Through appropriate scaling analyses they derived a reduced set of differential equations describing the structure of the Eulerian induced flow we examine herein. Although they presented the results of numerical simulations revealing the generation of induced flows, they did not obtain closed-form solutions nor did they perform a detailed analytic examination of the structure of the induced flows by two-dimensional wavepackets as it is influenced by rotation.

The generation of long waves can be understood to arise as a consequence of a localized translating source (i.e. the wavepacket) resulting from the divergence of the vertical flux of horizontal momentum. The vertical wavelength of the long waves is set by the vertical scale, $\ell_{z}$, of modulation of the waves. The frequency of the long waves is set by the forcing frequency, given by the ratio of the vertical group velocity, $c_{g z}$, to $\ell_{z}$ (§III.C of van den Bremer $\&$ Sutherland $\left.^{5}\right)$. Thus it is anticipated that the long wave response becomes evanescent if $c_{g z} / \ell_{z} \lesssim f$. This straightforward physical argument is consistent with the analysis of Tabaei \& Akylas ${ }^{4}$, who showed that rotation should play as important a role as wavepacket 
modulation upon induced flows if $f$ is scaled with $\ell_{z}^{-1}$.

The main purpose of the work presented here is to provide an explicit analytic expression for the flow induced by two-dimensional internal wavepackets in the presence of Coriolis forces. This is done in $\S I I$. The resulting expression permits analyses predicting the relative importance of induced long waves versus evanescent flows. Although we do not consider the weakly nonlinear feedback of the order amplitude-squared induced flows acting to Doppler-

shift waves within the wavepacket, we nonetheless examine the change in the structure of the induced mean flow over the extent of the wavepacket, as this should influence the modulational stability of the wavepacket ${ }^{4}$. In $§ I I I$ we present the results of numerical simulations, for comparison with the theoretical predictions. We discuss the relevance of our findings to oceanic and atmospheric applications in $\S$ ad conclusions are drawn in $\S \mathrm{V}$.

\section{THEORY}

Here we formulate the prediction for the flows induced by a quasi-monochromatic, twodimensional (i.e. spanwise-uniform), vertically propagating internal wavepacket. In doing so, we follow the approach of van den Bremer and Sutherland ${ }^{5}$ and extend their work to predict the Eulerian flow induced by vertically propagating two-dimensional internal wavepackets in uniformly stratified, Boussinesq fluid on the $f$-plane. The effects of viscosity and diffusion are neglected.

\section{A. Equations of motion}

The equations of motion for a two-dimensional, incompressible, Boussinesq, rotating fluid are

$$
\begin{aligned}
u_{x}+w_{z} & =0, \\
u_{t}+u u_{x}+w u_{z}-f v & =-P_{x}, \\
v_{t}+u v_{x}+w v_{z}+f u & =0 \\
w_{t}+u w_{x}+w w_{z} & =-P_{z}+b, \\
b_{t}+u b_{x}+w b_{z} & =-N^{2} w .
\end{aligned}
$$

Here $u, v$ and $w$ are the $x$-, $y$ - and $z$-components of velocity, $P \equiv p / \rho_{0}$ is the dynamic pressure divided by the characteristic density $\rho_{0}, b=-g \rho / \rho_{0}$ is the buoyancy cast in terms of the fluctuation density $\rho, g$ is gravity, $f$ is the (constant) Coriolis parameter and $N^{2}=$ $-\left(g / \rho_{0}\right) d \bar{\rho} / d z$ is the (constant) squared buoyancy frequency given in terms of the uniform background density gradient $d \bar{\rho} / d z$. Assuming spanwise uniform motion, the basic-state fields are independent of $y$, being functions only of the horizontal $x$-direction, the vertical, $z$, and time, $t$.

The equations may be combined by taking the curl of $(1 \mathrm{~b}, \mathrm{~d})$, which eliminates the pressure gradient terms, using (1a) to define the streamfunction, $\psi$, such that

$$
u=-\partial \psi / \partial z \text { and } w=\partial \psi / \partial x
$$

and then eliminating the linear differential operators acting on $b$ using (1e). The resulting evolution equation for $\psi$ is written with linear terms on the left-hand side and nonlinear 
terms on the right-hand side as follows:

$$
\underbrace{\left[\partial_{t t}\left(\partial_{x x}+\partial_{z z}\right)+N^{2} \partial_{x x}+f^{2} \partial_{z z}\right]}_{\equiv L} \psi=\nabla \cdot \underbrace{\left[\partial_{t}(\zeta \mathbf{u})+N^{2} \partial_{x}(\xi \mathbf{u})+f \partial_{z}(v \mathbf{u})\right]}_{\equiv \mathbf{F}} .
$$

Here we have denoted the spanwise vorticity by

$$
\zeta \equiv(\nabla \times \mathbf{u}) \cdot \hat{\mathbf{y}}=-\nabla^{2} \psi
$$

and buoyancy has been recast in terms of the vertical displacement

$$
\xi=-\rho /(d \bar{\rho} / d z)=-b / N^{2} .
$$

Equation (3) reduces to equation (30) of van den Bremer and Sutherland ${ }^{5}$ by taking $f=0$. Like their expression, the evolution equation is written as a linear differential operator, $L$, acting on $\psi$ being driven by nonlinear forcing expressed as the divergence of $\mathbf{F}$. With the inclusion of Coriolis forces, closure requires the inclusion of the evolution equation for the spanwise velocity $v$, given by (1c).

\section{B. Polarization relations}

We set out to find approximate solutions to (3) using perturbation theory for smallamplitude quasi-monochromatic internal wavepackets. This perturbation procedure involves the small parameter $\alpha$, which is a non-dimensional measure of the maximum amplitude of the wavepacket, and the small bandwidth parameter $\epsilon$, which is a measure of the wavelength relative to the length scale associated with modulation of the amplitude envelope of the wavepacket. For simplicity, we assume that the length scale for modulation in the $x$ - and $z$-directions are of comparable order.

Somewhat arbitrarily, we assume that the $O\left(\alpha \epsilon^{0}\right)$ vertical displacement field associated with the wavepacket is specified exactly by

$$
\xi_{0}^{(1)}=A(X, Z, T) e^{\imath \varphi} \text {, with } \varphi \equiv k x+m z-\omega t,
$$

in which it is understood that the actual vertical displacement field is the real part of this expression. The subscript on $\xi$ denotes the order of $\epsilon$ and the superscript denotes the order of $\alpha$. The quantity $A$ denotes the amplitude envelope of the wavepacket, which varies on the slow space and time scales, $(X, Z)$ and $T$, respectively. The envelope contains waves with wavenumber $\mathbf{k}=(k, m)$ and frequency $\omega=\omega(\mathbf{k})$. Thus we define $\alpha \sim|\mathbf{k}||| A \| \ll 1$ and $\epsilon \sim\left\|A_{X}\right\| /(|\mathbf{k}|\|A\|) \sim\left\|A_{Z}\right\| /(|\mathbf{k}|\|A\|) \ll 1$. It is convenient to consider the wavepacket evolution in a frame of reference moving at the group velocity, $\mathbf{c}_{g}=\nabla_{\mathbf{k}} \omega$, of the wavepacket so that

$$
X=x-c_{g x} t \quad \text { and } \quad Z=z-c_{g z} t .
$$

In this frame, the temporal evolution represented by the dependence of the amplitude envelope upon $T$, describes the dispersion of the wavepacket, which is order $\epsilon^{2}$, and so will be negligible at the orders of perturbation theory considered below. 


\begin{tabular}{lll} 
Field & $O\left(\alpha \epsilon^{0}\right)$ & $O\left(\alpha \epsilon^{1}\right)$ \\
\hline Vertical displacement $(\xi)$ & $A$ & 0 \\
Streamfunction $(\psi)$ & $-\frac{\omega}{k} A$ & $-\imath \frac{\omega}{k}\left[\frac{1}{k}(1-C) \partial_{X} A+\frac{1}{m} C \partial_{Z} A\right]$ \\
Along-stream velocity $(u)$ & $\imath \frac{\omega m}{k} A$ & $\frac{\omega m}{k}(1-C)\left[-\frac{1}{k} \partial_{X} A+\frac{1}{m} \partial_{Z} A\right]$ \\
Spanwise velocity $(v)$ & $\frac{f m}{k} A$ & $\imath \frac{f m}{k}\left[\frac{1}{k} \partial_{X} A-\frac{1}{m} \partial_{Z} A\right]$ \\
Vertical velocity $(w)$ & $-\imath \omega A$ & $\omega C\left[-\frac{1}{k} \partial_{X} A+\frac{1}{m} \partial_{Z} A\right]$ \\
Vorticity $(\zeta)$ & $-\frac{\omega|\mathbf{k}|^{2}}{k} A$ & $\imath \frac{\omega|\mathbf{k}|^{2}}{k}\left[\frac{1}{k}\left(\frac{N^{2} k^{4}-f^{2} m^{4}}{\omega^{2}|\mathbf{k}|^{4}}\right) \partial_{X} A+\right.$ \\
& & $\left.\frac{1}{m}\left(C+2 \frac{f^{2} m^{2}}{\omega^{2}|\mathbf{k}|^{2}}\right) \partial_{Z} A\right]$
\end{tabular}

TABLE I. Coefficients of $e^{\imath \varphi} \equiv \exp [\imath(k x+m z-\omega t)]$ in the polarization relations corresponding to indicated fields evaluated at $O\left(\alpha \epsilon^{0}\right)$ (second column) and $O(\alpha \epsilon)$ (third column) given in terms of the amplitude envelope $A$ of the vertical displacement field. It is understood that the actual fields are given by the real parts of the tabulated expressions after multiplication by $e^{\imath \varphi}$. In the above expressions, $C \equiv k^{2} m^{2}\left(N^{2}-f^{2}\right) /\left(\omega^{2}|\mathbf{k}|^{4}\right)$.

Taking terms at $O\left(\alpha \epsilon^{0}\right)$ in (3) gives the standard dispersion relation for internal waves that are influenced by rotation:

$$
\omega^{2}=\left(N^{2} k^{2}+f^{2} m^{2}\right) /\left(k^{2}+m^{2}\right) .
$$

Likewise, the polarization relations at this order can be found from the linearized equations of motion assuming plane wave solutions. These are listed in the second column of Table I. For what follows, it is necessary to evaluate the corrections to the polarization relations at $O(\alpha \epsilon)$, expressions that involve single spatial derivatives of the amplitude envelope, $A$. These are listed in the third column of Table I.

\section{Nonlinear forcing}

Given the polarization relations in Table I, we can proceed to compute the nonlinear terms on the right-hand side of (3) to leading order. Specifically, we seek only the forcing terms on the wavepacket scale, neglecting superharmonic terms proportional to $\exp ( \pm 2 \imath \varphi)$. Thus, for example, from the correlation $\zeta u$ in the first term of $\mathbf{F}$ in (3), we consider only the leading-order forcing $\overline{\zeta u}=\overline{\zeta^{(1)} u^{(1)}}$, where the over-bar denotes averaging over the fast wave scales. All of these correlations, listed in Table II, are $O\left(\alpha^{2} \epsilon\right)$ at leading order, involving single spatial derivatives of $|A|^{2}$.

Combining these results, we see that the leading-order forcing on the right-hand side of (3) is $O\left(\alpha^{2} \epsilon^{3}\right)$. Explicitly, the forcing can be expressed as

$$
(\nabla \cdot \mathbf{F})_{3}^{(2)}=-\frac{N^{4} k^{3} m^{2}}{2 \omega|\mathbf{k}|^{6}}\left(C_{X X X} \partial_{X X X}+C_{X X Z} \partial_{X X Z}+C_{X Z Z} \partial_{X Z Z}+C_{Z Z Z} \partial_{Z Z Z}\right)|A|^{2},
$$




\begin{tabular}{ll} 
Correlation & Value \\
\hline$(\overline{\overline{\zeta u}})_{1}^{(2)}$ & $\frac{1}{4}\left(\omega^{2} / k^{2}\right)\left[2 k m \partial_{X}+\left(m^{2}-k^{2}\right) \partial_{Z}\right]|A|^{2}$ \\
$(\overline{\zeta w})_{1}^{(2)}$ & $\frac{1}{4}\left(\omega^{2} / k^{2}\right)\left[\left(m^{2}-k^{2}\right) \partial_{X}-2 k m \partial_{Z}\right]|A|^{2}$ \\
$(\overline{\xi u})_{1}^{(2)}$ & $\frac{1}{4} \frac{\omega}{k^{2}}(1-C)\left[-m \partial_{X}+k \partial_{Z}\right]|A|^{2}$ \\
$(\overline{\xi w})_{1}^{(2)}$ & $\frac{1}{4} \frac{\omega}{k m} C\left[-m \partial_{X}+k \partial_{Z}\right]|A|^{2}$ \\
$(\overline{v u})_{1}^{(2)}$ & $\frac{1}{4} \frac{\omega f m}{k^{3}} C\left[m \partial_{X}-k \partial_{Z}\right]|A|^{2}$ \\
$(\overline{v w})_{1}^{(2)}$ & $\frac{1}{4} \frac{\omega f}{k^{2}}(1+C)\left[-m \partial_{X}+k \partial_{Z}\right]|A|^{2}$
\end{tabular}

TABLE II. Slowly varying parts of the correlations appearing in the leading-order nonlinear forcing terms on the right-hand side of (3).

in which the coefficients are given by

$$
\begin{aligned}
C_{X X X} & =\frac{m}{k}\left(\frac{1}{2 \gamma}(1+3 \gamma)+\frac{1}{2} \Delta(5+\gamma)-\Delta^{2} \gamma\right), \\
C_{X X Z} & =-\frac{1}{2 \gamma}\left(1+4 \gamma-3 \gamma^{2}\right)+\frac{1}{2} \Delta(1-11 \gamma)+\frac{\gamma}{2} \Delta^{2}(5-\gamma), \\
C_{X Z Z} & =\frac{m}{k}\left(\frac{1}{2 \gamma}(1-5 \gamma)+\frac{1}{2} \Delta(11-\gamma)-\frac{1}{2} \Delta^{2}\left(3-4 \gamma-\gamma^{2}\right)\right), \\
C_{Z Z Z} & =1-\Delta \frac{1}{2 \gamma}(1+5 \gamma)-\Delta^{2} \frac{\gamma}{2}(3+\gamma),
\end{aligned}
$$

in which $\Delta \equiv(f / N)^{2}$ and $\gamma \equiv(m / k)^{2}$.

\section{Eulerian induced flow}

As originally found by Bretherton ${ }^{3}$, two-dimensional internal wavepackets in the absence of rotation induce an Eulerian flow that is manifest as horizontally long internal waves emanating outward and leeward of the wavepacket (for example, see Fig. 5 of van den Bremer and Sutherland ${ }^{5}$ ). The long wave response to localized forcing inspires the simplification of the right-hand side forcing in (3) originally made by Bretherton ${ }^{3}$ such that

$$
(\nabla \cdot \mathbf{F})_{3}^{(2)} \simeq \mathcal{N}(Z) \delta(k X),
$$

in which

$$
\mathcal{N}(Z)=k \int_{-\infty}^{\infty}(\nabla \cdot \mathbf{F})_{3}^{(2)} d X=-\frac{N^{4} k^{4} m^{2}}{2 \omega|\mathbf{k}|^{6}} C_{Z Z Z} \int_{-\infty}^{\infty} \partial_{Z Z Z}|A|^{2} d X
$$

Hence all terms in (10) vanish except for that involving $Z$-derivatives alone. That term arises from the correlations $\overline{\zeta w}$ and $\overline{v w}$.

In particular, if the amplitude envelope of the vertical displacement field is separable such that $A(X, Z)=A_{0} \eta(X) \mathcal{A}(Z)$ with $\|\eta\|=\|\mathcal{A}\|=1$, then (12) gives

$$
\mathcal{N}(Z)=-A_{0}^{2} \ell_{x} \frac{N^{4} k^{4} m^{2}}{2 \omega|\mathbf{k}|^{6}} C_{Z Z Z} \frac{d^{3}}{d Z^{3}}|\mathcal{A}|^{2},
$$

in which $\ell_{x} \equiv \int_{-\infty}^{\infty} \eta^{2}(X) d X$. In the special case of the wavepacket being a Gaussian in the horizontal such that $\eta(X)=\exp \left[-X^{2} /\left(2 \sigma_{x}^{2}\right)\right]$, then $\ell_{x}=\sigma_{x} \sqrt{\pi}$. 
To find the induced flow that arises in response to this forcing at $O\left(\alpha^{2}\right)$, we manipulate the linear terms on the left-hand side of (3), re-expressing the derivatives in terms of the translating co-ordinates $(X, Z)$ and neglecting the partial $X$ derivatives in the Laplacian and higher-order time derivative terms. The former is a consequence of assuming the response is wider than the wavepacket in the $x$-direction as for the case without Coriolis forces. Hence the leading-order equation for the induced Eulerian flow in terms of the $O\left(\alpha^{2}\right)$ streamfunction $\psi^{(2)}$ is

$$
\left(c_{g z}^{2} \partial_{Z Z Z Z}+N^{2} \partial_{X X}+f^{2} \partial_{Z Z}\right) \psi^{(2)}=\mathcal{N}(Z) \delta(k X) .
$$

The solution is found in Fourier space by writing

$$
\psi^{(2)}(X, Z)=\iint \hat{\psi}(\kappa, \mu) e^{\imath(\kappa X+\mu Z)} d \kappa d \mu,
$$

and likewise transforming the forcing given by (11) with (12). Using (13), the resulting algebraic equation for $\hat{\psi}$ is solved to give

$$
\hat{\psi}=\imath A_{0}^{2} \ell_{x} \frac{N^{4} k^{3} m^{2}}{4 \pi \omega|\mathbf{k}|^{6}} C_{Z Z Z} \frac{\mu^{3} \widehat{\left.\mathcal{A}\right|^{2}}}{c_{g z}^{2} \mu^{4}-N^{2} \kappa^{2}-f^{2} \mu^{2}},
$$

in which $\widehat{|\mathcal{A}|^{2}}(\mu)$ is the Fourier transform of $|\mathcal{A}|^{2}(Z)$.

We seek an analytic solution in real space as a single integral through identifying singularities in the inverse Fourier transform of (16). Following van den Bremer \& Sutherland ${ }^{5}$, the denominator in (16) is factored in the following way:

$$
\frac{1}{c_{g z}^{2} \mu^{4}-f^{2} \mu^{2}-N^{2} \kappa^{2}}=\frac{1}{2 c_{g z} \mu \sqrt{\mu^{2}-\mu_{0}^{2}}}\left[\frac{1}{c_{g z} \mu \sqrt{\mu^{2}-\mu_{0}^{2}}-N \kappa}+\frac{1}{c_{g z} \mu \sqrt{\mu^{2}-\mu_{0}^{2}}+N \kappa}\right],
$$

in which we have defined $\mu_{0} \equiv\left|f / c_{g z}\right|$. From this, we can perform the integration in $\kappa$ explicitly. However, we see that when inverting the Fourier transform, we must separately consider cases with $|\mu|>\mu_{0}$ (yielding singularities on the real $\kappa$-axis) and $|\mu|<\mu_{0}$ (yielding singularities on the imaginary $\kappa$-axis). For the real singularities, we obtain long wave solutions by taking a branch cut corresponding to outgoing waves. For the imaginary singularities, we obtain evanescent wave solutions using the residue theorem, enforcing boundedness of the solutions. Thus we arrive at the following:

$$
\begin{aligned}
\psi^{(2)}=-\frac{1}{2} A_{0}^{2} \ell_{x} \frac{N^{3} k m}{\left(N^{2}-f^{2}\right)|\mathbf{k}|^{2}} C_{Z Z Z}[ & \int_{\mu_{0}}^{\infty} \frac{\mu^{2} \mid \widehat{\left.\mathcal{A}\right|^{2}}}{\sqrt{\mu^{2}-\mu_{0}^{2}}} \cos \left(\frac{c_{g z}}{N} \mu \sqrt{\mu^{2}-\mu_{0}^{2}}|X|+\mu Z\right) d \mu \\
& \left.+\int_{0}^{\mu_{0}} \frac{\mu^{2} \mid \widehat{\left.\mathcal{A}\right|^{2}}}{\sqrt{\mu_{0}^{2}-\mu^{2}}} \exp \left(\frac{-c_{g z}}{N} \mu \sqrt{\mu_{0}^{2}-\mu^{2}}|X|\right) \sin (\mu Z) d \mu\right] .
\end{aligned}
$$

The first integral represents the induced flow manifest as long waves, while the second integral represents the induced flow manifest as evanescent waves occurring when the frequency 
associated with the induced flow is less than $f$. The corresponding expression for the horizontal wave induced mean flow is

$$
\begin{aligned}
u^{(2)}=-\frac{1}{2} A_{0}^{2} \ell_{x} \frac{N^{3} k m}{\left(N^{2}-f^{2}\right)|\mathbf{k}|^{2}} C_{Z Z Z}[ & \int_{\mu_{0}}^{\infty} \frac{\mu^{3} \mid \widehat{\left.\mathcal{A}\right|^{2}}}{\sqrt{\mu^{2}-\mu_{0}^{2}}} \sin \left(\frac{c_{g z}}{N} \mu \sqrt{\mu^{2}-\mu_{0}^{2}}|X|+\mu Z\right) d \mu \\
& \left.-\int_{0}^{\mu_{0}} \frac{\mu^{3} \mid \widehat{\left.\mathcal{A}\right|^{2}}}{\sqrt{\mu_{0}^{2}-\mu^{2}}} \exp \left(-\frac{c_{g z}}{N} \mu \sqrt{\mu_{0}^{2}-\mu^{2}}|X|\right) \cos (\mu Z) d \mu\right] .
\end{aligned}
$$

This may be written as $u^{(2)}=u_{\mathrm{lw}}+u_{\mathrm{ev}}$, corresponding to the sum of the induced flow associated with long waves (the first integral with $\mu>\mu_{0}$ ) and evanescent disturbances (the second integral with $\left.0<\mu<\mu_{0}\right)$.

We can find the induced spanwise flow, $v^{(2)}$, from the $y$-momentum equation (1c) written in the translating $(X, Z)$ co-ordinates:

$$
-\left(c_{g x} \partial_{X}+c_{g z} \partial_{Z}\right) v^{(2)}=-\partial_{X}\left(\overline{v^{(1)} u^{(1)}}\right)-\partial_{Z}\left(\overline{v^{(1)} w^{(1)}}\right)+f \partial_{Z}\left(\psi^{(2)}\right) .
$$

The induced flow varies more slowly in $X$ compared with $Z$, and so the $c_{g x} \partial_{X}$ term on the left-hand side can be neglected. Furthermore, the advective terms are smaller than the last term on the right-hand side. Thus the dominant contribution to $v^{(2)}$ is given simply by

$$
v^{(2)}=-\mu_{0} \psi^{(2)}
$$

The buoyancy field associated with the Eulerian induced flow is likewise found by writing the buoyancy equation (1e) in translating co-ordinates and neglecting the $c_{g x} \partial_{X}$ term and the relatively small advective terms. The equation for $b^{(2)}$ thus satisfies

$$
\partial_{Z} b^{(2)}=\left(N^{2} / c_{g z}\right) \partial_{X} \psi^{(2)}
$$

The induced flow predicted by (18) is shown for three cases in Figures 1 - 3. In all cases the upward-propagating wavepacket has a bivariate Gaussian amplitude envelope given by $A(X, Z)=A_{0} \exp \left[-\left(X^{2} / \sigma_{x}^{2}+Z^{2} / \sigma_{z}^{2}\right) / 2\right]$, in which $\sigma_{x}=\sigma_{z}=20 / k$. Figure 1 shows the case in which the waves contained in the packet have $m=-k$. In the non-rotating case $(f=0)$, the induced flow has the well-known form of the long-wave response ${ }^{3-5}$. This result can be compared with the total induced flow in the case $f=0.01 N$ (Fig. 1b). The amplitude and structure of the long wave response are similar to those in the non-rotating case except that there is also a signal of horizontally uniform, vertically periodic waves contained between the outward propagating induced long waves. The total induced flow is decomposed into its contributions from $u_{\mathrm{lw}}$ and $u_{\mathrm{ev}}$, as shown in Figs. $1 \mathrm{c}$ and $1 \mathrm{~d}$, respectively. Both components involve horizontally uniform, vertically periodic disturbances both above and below the wavepacket. However, the superposition of these fields result in destructive interference above the wavepacket and constructive interference below. Hence there is no disturbance by the total induced flow sufficiently far above the wavepacket and a weak vertically periodic disturbance below. We examine the asymptotic behaviour in more detail in $\S$ IIF. 

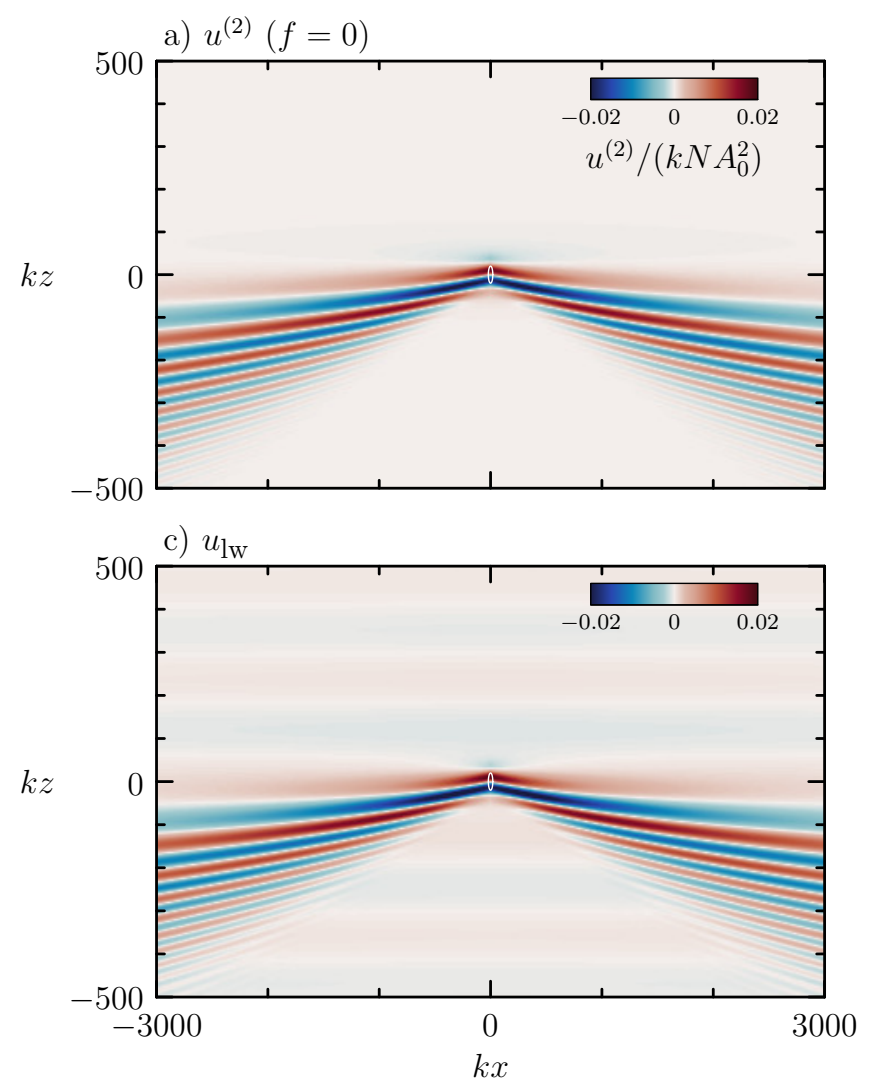
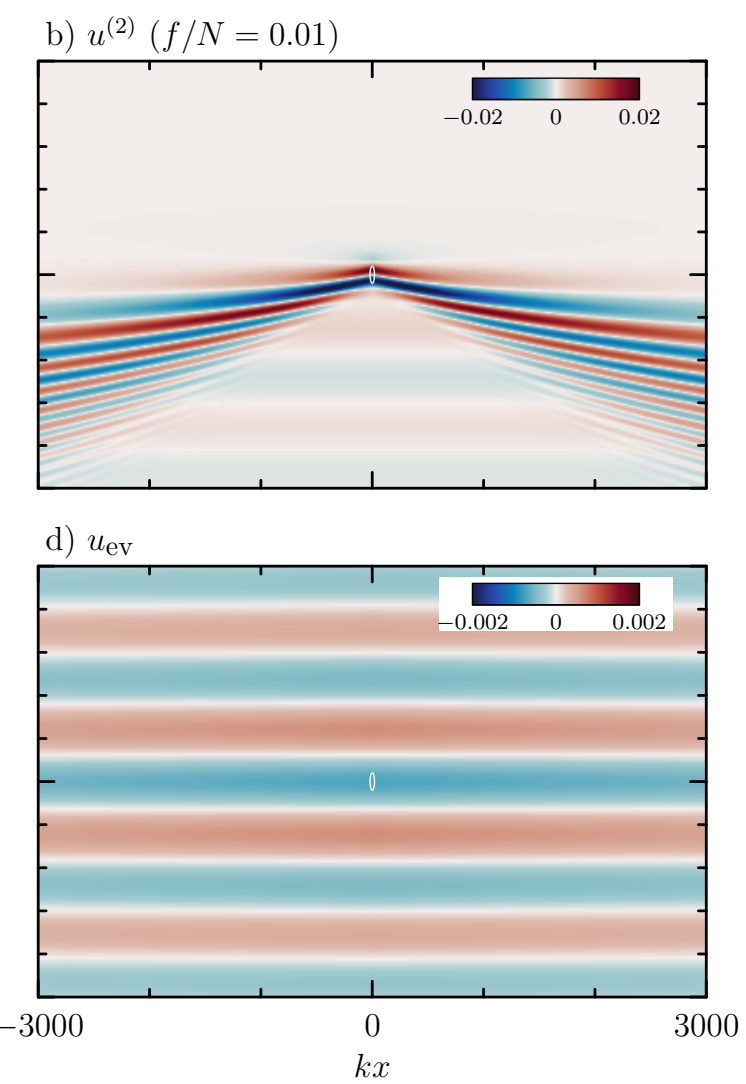

FIG. 1. Horizontal velocity associated with the wave-induced flow for a Gaussian wavepacket with $k \sigma_{x}=k \sigma_{z}=20$, containing waves with $m / k=-1$ : a) induced flow computed with $f=0, \mathrm{~b}$ ) induced flow computed with $f / N=0.01$ and its decomposition into the flow associated with c) long waves and d) evanescent disturbances. In all cases the velocity is normalized by $k N A_{0}^{2}$. The white circle centred at the origin (appearing as an ellipse in these stretched co-ordinates) is drawn with radius $20 / k$, indicating the spatial extent of the wavepacket. Note the reduced in scale in d) that serves to show better the relatively small evanescent disturbances.

For a wavepacket containing more hydrostatic waves with $m=-3 k$, the influence of rotation upon the Eulerian induced flow is significantly more pronounced, as shown in Figure 2. If there is no rotation, the long waves emanate downward to the right and left of the wavepacket at a shallower angle to the horizontal. This is consistent with the scaling argument that the frequency of the long waves should be of the order $c_{g z} / \ell_{z}$, and the fact that the vertical group velocity decreases as $|m / k|$ increases provided $|m / k| \gtrsim 1 / \sqrt{2}$. For the wavepacket with $m=-3 k$ and $f=0, \omega \simeq 0.32 N$ and $c_{g z} \simeq 0.095 N / k$. Hence, taking $\ell_{z}=\sigma_{z}$, the long waves have frequency on the order $c_{g z} / \ell_{z} \simeq 0.0047 N$. With a typical value of the relative Coriolis parameter, $f=0.01 N$, rotation has little influence upon waves in the wavepacket. However, a significant proportion of the spectrum of induced long waves is anticipated to be evanescent, as evident in Fig. 2b-d. In this case the contributions to the total flow from $u_{\mathrm{l}}$ and $u_{\mathrm{ev}}$ are comparable. While the long wave contribution has a signal of down- and outward propagating waves, both this and the evanescent contribution exhibit 

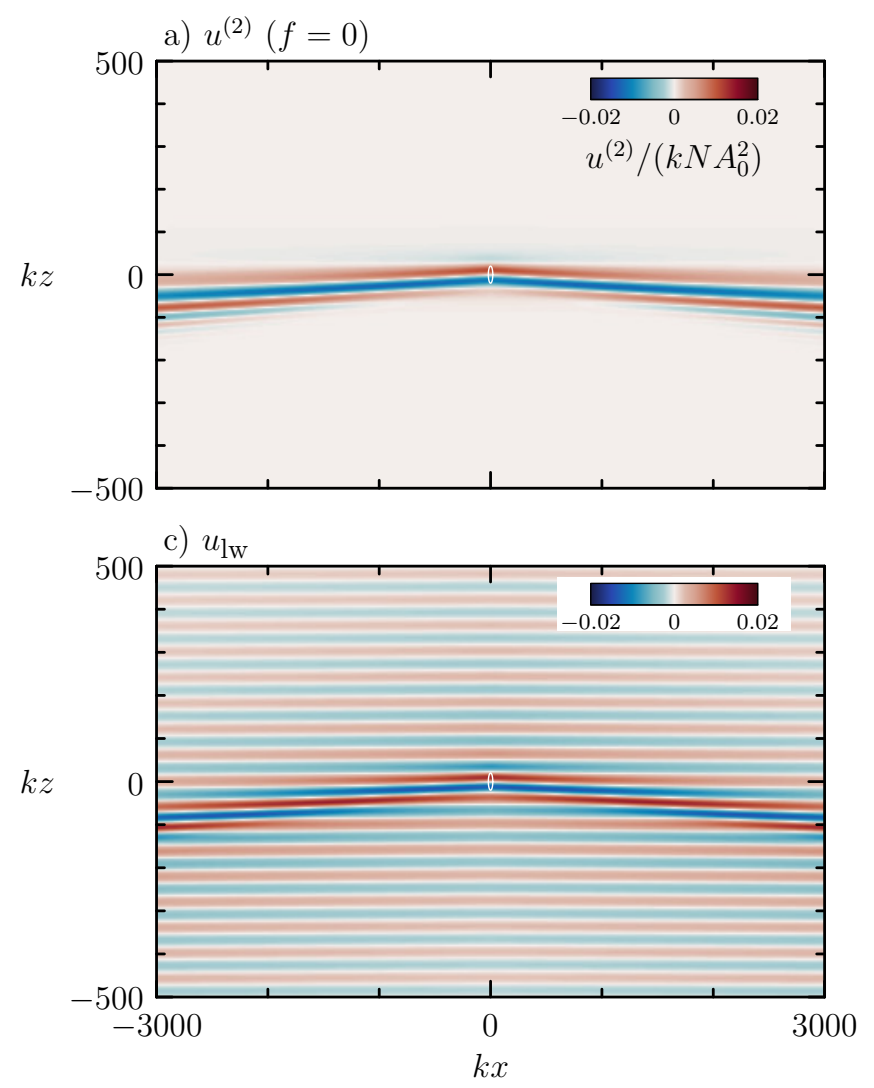
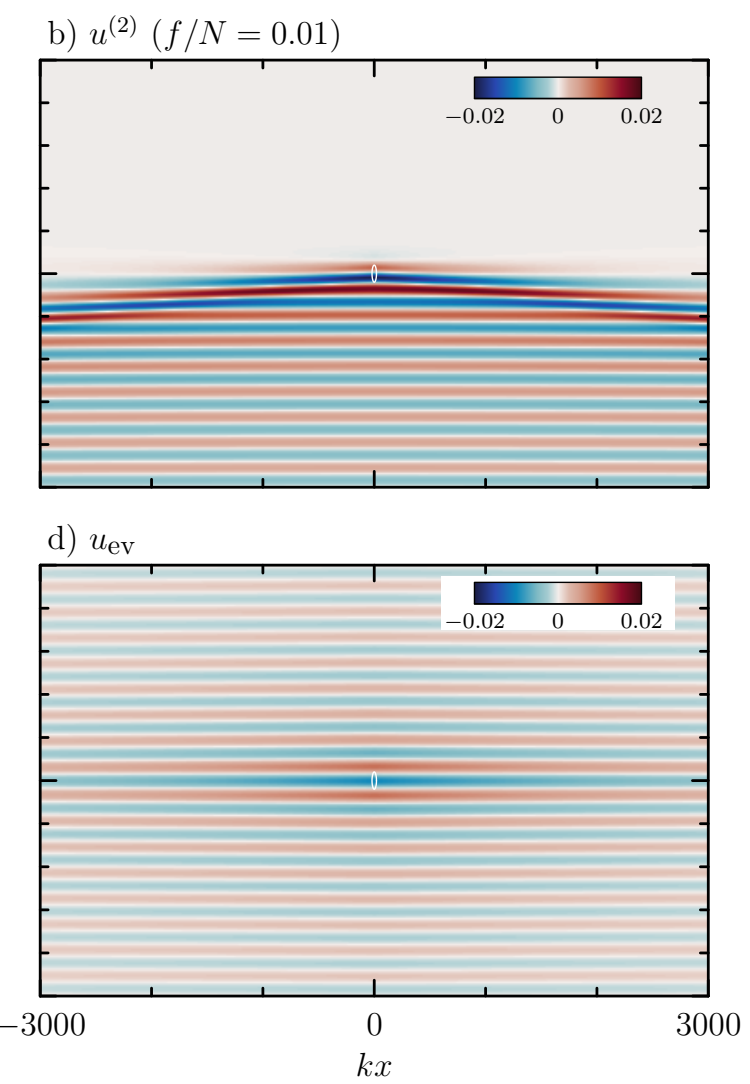

FIG. 2. As in Fig. 1 but for an internal wavepacket containing waves with $m / k=-3$.

horizontally uniform, vertical periodic disturbances as well. As in the case with $m=-k$, these disturbances destructively interfere above the wavepacket so that the total induced flow is non-negligible only below the wavepacket.

In the case with $m=-5 k$, shown in Fig. 3, the vertical group velocity of the wavepacket, $c_{g z} \simeq 0.038 \mathrm{~N} / k$, is so small that the long waves have frequency on the order $0.0019 \mathrm{~N}$. Correspondingly, although shallow long waves are evident in the case with $f=0$, the contribution from $u_{\mathrm{lw}}$ is negligible if $f=0.01 \mathrm{~N}$. In this case, the total induced flow, which is dominated by $u_{\mathrm{ev}}$, is characterised by horizontal disturbances that decay to the left and right of the wavepacket as well as above and below. The enhanced lateral confinement of the induced flow due to rotation was also noted in the simulations of Wilhelm et al ${ }^{20}$.

\section{E. Horizontally integrated flow}

It is insightful to consider the horizontal integral of the induced horizontal flow, $u^{(2)}$. After integrating in $X$, the two integrals in (18) combine and the result can be manipulated to cast it in the following form:

$$
\left.\int u^{(2)} d X=\ell_{x} \mathcal{C}\left[\int_{0}^{\infty} \frac{\mu^{2}}{\mu^{2}-\mu_{0}^{2}} \widehat{\left.\mathcal{A}\right|^{2}(\mu}\right) \cos (\mu Z) d \mu\right]=\ell_{x}\left(U_{\mathrm{DF}}(Z)+\bar{V}(Z)\right),
$$



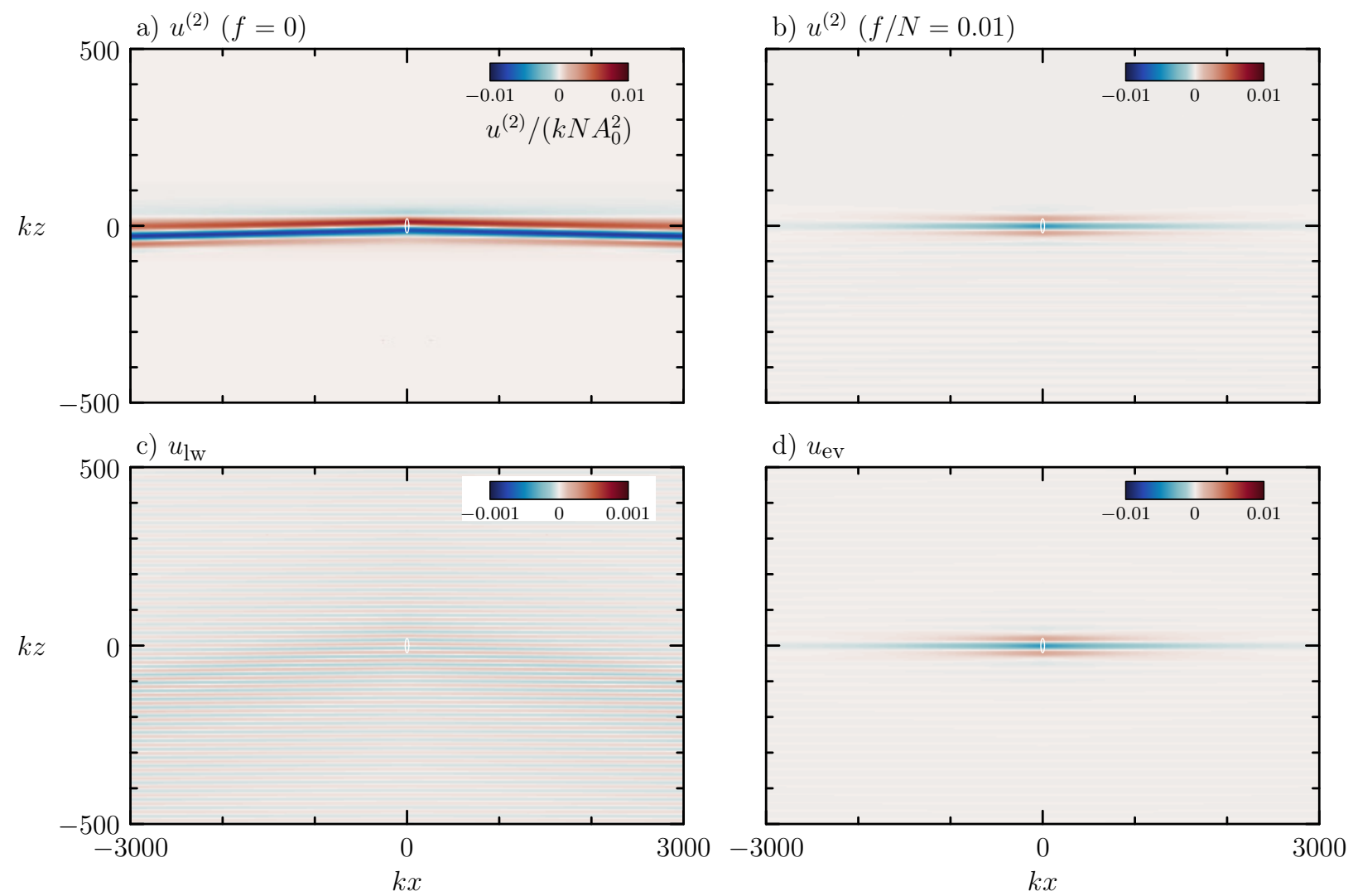

FIG. 3. As in Fig. 1 but for wavepacket containing waves with $m / k=-5$. Note the reduced in scale in c).

in which

$$
U_{\mathrm{DF}} \equiv \mathcal{C}|\mathcal{A}|^{2}(Z), \quad \bar{V} \equiv \mathcal{C} \int_{-\infty}^{\infty} \frac{\mu_{0}^{2}}{\mu^{2}-\mu_{0}^{2}} \mid \widehat{\left.\mathcal{A}\right|^{2}(\mu)} e^{i \mu Z} d \mu
$$

and

$$
\mathcal{C} \equiv \frac{1}{2} N|\mathbf{k}| A_{0}^{2} \frac{N^{4}}{\left(N^{2}-f^{2}\right)^{2}}\left(1+\frac{f^{2} m^{2}}{N^{2} k^{2}}\right)^{1 / 2} C_{Z Z Z}
$$

If $f=0$, then $\bar{V}=0$ and $U_{\mathrm{DF}}$ is equal to the divergent-flux induced flow $U_{\mathrm{DF} 0}$, found by horizontally averaging the horizontal momentum equation, assuming horizontally periodic waves $^{21}$ :

$$
U_{\mathrm{DF} 0}(Z) \equiv\left\langle u_{1} w_{1}\right\rangle / c_{g z}=\frac{1}{2} N|\mathbf{k}| A_{0}^{2}|\mathcal{A}(Z)|^{2} .
$$

For non-rotating two-dimensional wavepackets, the horizontal integral of the Eulerian induced flow, $u^{(2)}$, was shown to equal $U_{\mathrm{DF} 0} \ell_{x}$ in van den Bremer \& Sutherland ${ }^{5}$. In other words, all the horizontal momentum in $u^{(2)}$ originates from the divergent-flux induced flow of the wavepacket.

If rotation is not neglected, then the expression for $\bar{V}$ can be seen to result from the Coriolis term in the $x$-momentum equation (1b). Explicitly, $\ell_{x} \bar{V}=-\left(1 / c_{g z}\right) \int^{Z} \int_{-\infty}^{\infty} f v^{(2)}(X, \tilde{Z}) d X d \tilde{Z}$, in which $v^{(2)}$ is given by (19). In other words, not all the horizontal momentum in $u^{(2)}$ originates from the divergent-flux induced flow of the wavepacket. 


\section{F. Near and far-field asymptotics}

To get further insight into the structure of the induced flow field, we consider the flow at the centre of wavepacket as well as far above and below the wavepacket at $X=0$.

\section{Near-field asymptotics}

Starting with the near field, performing a Taylor series expansion in $Z$, we obtain for small $Z$ at $X=0$

$$
\left.u^{(2)}\right|_{X=0} \simeq-\frac{1}{2} A_{0}^{2} \ell_{x} \frac{N^{3} k m}{\left(N^{2}-f^{2}\right)|\mathbf{k}|^{2}} C_{Z Z Z} \frac{1}{\ell_{z}^{2}} \sum_{n=0}^{\infty} C_{n}\left(\frac{Z}{\ell_{z}}\right)^{n}, \quad|Z| / \ell_{z} \ll 1,
$$

where $\ell_{z}$ represents the characteristic vertical scale of the modulation to the wavepacket in $Z$, and the nondimensional coefficients, $C_{n}$, are given by

$$
\begin{aligned}
& C_{2 j}=\frac{(-1)^{j+1}}{(2 j) !} \int_{0}^{\tilde{\mu}_{0}} \frac{\tilde{\mu}^{2 j+3}}{\sqrt{\tilde{\mu}_{0}^{2}-\tilde{\mu}^{2}}} \frac{1}{\ell_{z}} \mid \widehat{\left.\mathcal{A}\right|^{2}(\tilde{\mu})} d \tilde{\mu}, \quad j=0,1,2, \ldots, \\
& C_{2 j+1}=\frac{(-1)^{j}}{(2 i+1) !} \int_{\tilde{\mu}_{0}}^{\infty} \frac{\tilde{\mu}^{2 i+4}}{\sqrt{\tilde{\mu}^{2}-\tilde{\mu}_{0}^{2}}} \frac{1}{\ell_{z}} \mid \widehat{\left.\mathcal{A}\right|^{2}(\tilde{\mu})} d \tilde{\mu}, \quad j=0,1,2, \ldots,
\end{aligned}
$$

in which $\tilde{\mu}_{0} \equiv \mu_{0} \ell_{z}$. For a Gaussian wavepacket we set $\ell_{z}=\sigma_{z}$, in which case $\mid \widehat{\left.\mathcal{A}\right|^{2}(\tilde{\mu})} / \ell_{z}=$ $\exp \left(-\tilde{\mu}^{2} / 4\right) /(2 \sqrt{\pi})$. The corresponding integrals in (26) can be evaluated numerically, and are plotted in Fig. 4 for $n=0,1,2$ and 3. For small $\tilde{\mu}_{0}$, the leading coefficients are

$$
\begin{gathered}
C_{0} \simeq-\frac{1}{3 \sqrt{\pi}} \tilde{\mu}_{0}^{3}, \quad C_{2} \simeq \frac{2}{15 \sqrt{\pi}} \tilde{\mu}_{0}^{5} \\
C_{1} \simeq \frac{4}{\sqrt{\pi}}\left(1+\tilde{\mu}_{0}^{2} / 8\right), \quad C_{3} \simeq-\frac{16}{3 \sqrt{\pi}}\left(1+9 \tilde{\mu}_{0}^{2} / 128\right) .
\end{gathered}
$$

The corresponding asymptotic approximations for large $\tilde{\mu}_{0}$ are

$$
\begin{gathered}
C_{0} \simeq-\frac{4}{\sqrt{\pi}} \frac{1}{\tilde{\mu}_{0}}\left(1+4 \tilde{\mu}_{0}^{-2}\right), \quad C_{2} \simeq \frac{16}{\sqrt{\pi}} \frac{1}{\tilde{\mu}_{0}}\left(1+6 \tilde{\mu}_{0}^{-2}\right), \\
C_{1} \simeq \frac{1}{2} \tilde{\mu}_{0}^{3} \exp \left(-\tilde{\mu}_{0}^{2} / 4\right)\left[1+3 \tilde{\mu}_{0}^{-2}\right], \quad C_{3} \simeq-\frac{1}{12} \tilde{\mu}_{0}^{5} \exp \left(-\tilde{\mu}_{0}^{2} / 4\right)\left[1+5 \tilde{\mu}_{0}^{-2}\right] .
\end{gathered}
$$

These are shown by dotted lines in Fig. 4. The coefficients with odd indices (plotted in blue) come from the first integral in (18), which corresponds to induced long waves, whereas the coefficients with even indices (plotted in black) come from the second integral corresponding to evanescent disturbances. The plots show a transition from horizontal flows over the wavepacket that are odd in $Z$ for $\tilde{\mu}_{0} \ll 1$ (when dominated by long waves) to flows that are even in $Z$ for $\tilde{\mu}_{0} \gg 1$ (when dominated by evanescent disturbances). To be more precise, the maximum absolute values of the horizontal flows associated with the long waves and evanescent disturbances are equal for $\tilde{\mu}_{0} \sim \tilde{\mu}_{0}^{\star}=2.4$. Hence, long waves dominate if $\tilde{\mu}_{0}<\tilde{\mu}_{0}^{\star}$ and evanescent disturbances dominate otherwise. The corresponding asymptotic curves are superimposed on the exact solution for $u^{(2)}(X=0, Z)$ in Fig. 5. 

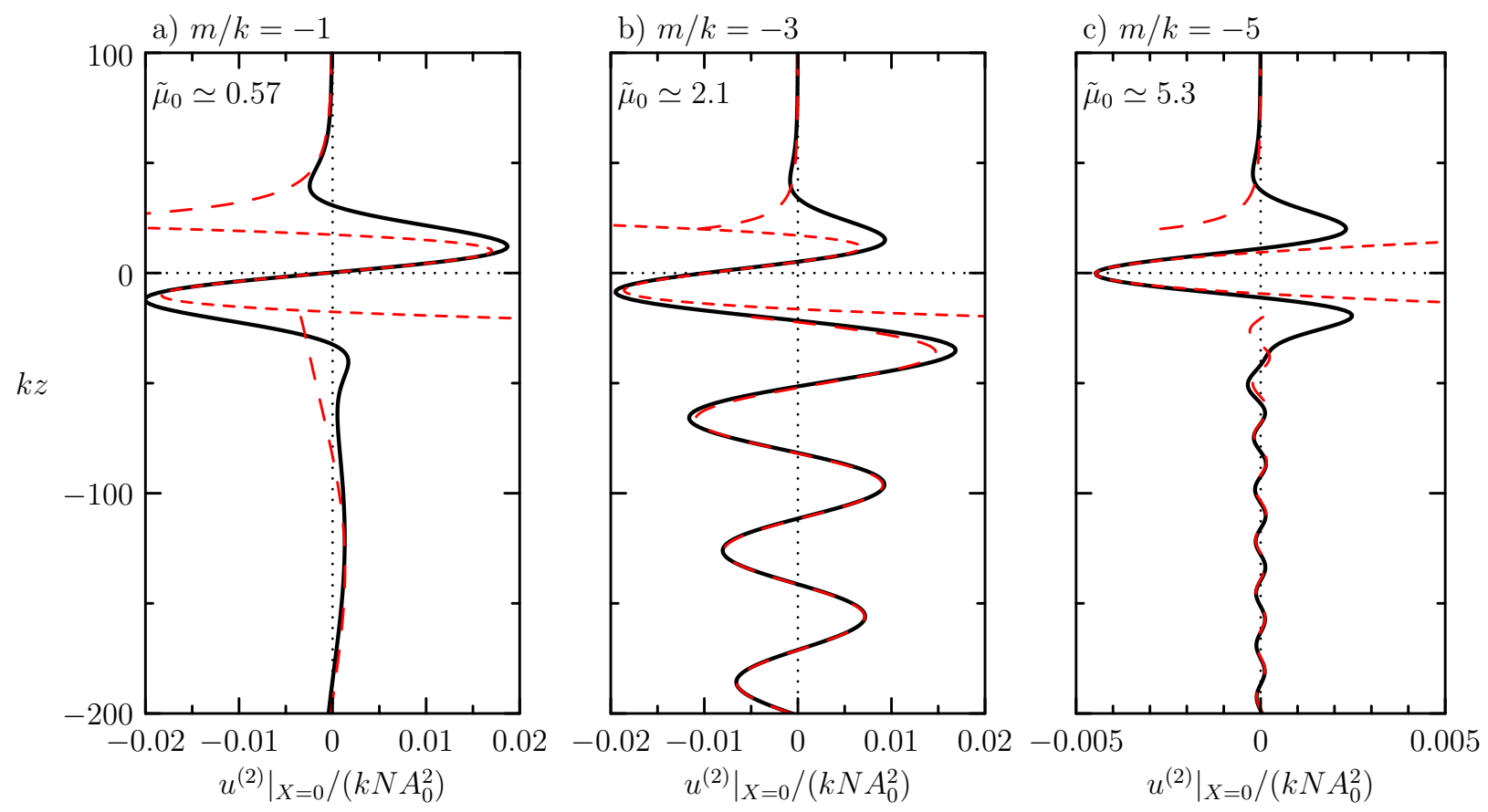

FIG. 4. First 4 coefficients $C_{n}\left(\tilde{\mu}_{0}\right)$ used in the approximation to $u^{(2)}(X=0, Z)$ for $\left|Z / \sigma_{z}\right| \ll 1$, as defined by (26) for a Gaussian wavepacket.

\section{Far-field asymptotics}

Using the method of steepest descents upon the integrals in (18), we find for the far field

$\left.u^{(2)}\right|_{X=0} \simeq-\frac{1}{2} A_{0}^{2} \ell_{x} \frac{N^{3} k m}{\left(N^{2}-f^{2}\right)|\mathbf{k}|^{2}} C_{Z Z Z} \mu_{0}^{3} \begin{cases}-\widehat{|\mathcal{A}|^{2}(0)} \frac{6}{\left(\mu_{0} Z\right)^{4}} & Z / \ell_{z} \rightarrow \infty, \\ \mid \widehat{\left.\mathcal{A}\right|^{2}\left(\mu_{0}\right)} \sqrt{\frac{2 \pi}{\left|\mu_{0} Z\right|}} \sin \left(\mu_{0} Z-\pi / 4\right) & Z / \ell_{z} \rightarrow-\infty .\end{cases}$

For the bivariate Gaussian, $\ell_{x}=\sqrt{\pi} \sigma_{x}$ and $\widehat{|\mathcal{A}|^{2}}(\mu)=\sigma_{z} /(2 \sqrt{\pi}) \exp \left(-\mu^{2} \sigma_{z}^{2} / 4\right)$. The corresponding asymptotic curves are superimposed on the exact solution for $u^{(2)}(X=0, Z)$ in Fig. 5. The result clearly shows that, under the influence of rotation, the induced flow is manifest as a vertically decaying oscillating disturbance below the wavepacket whose wavelength decreases as $\mu_{0}=f / c_{g z}$ increases. Despite the factor $\mu_{0}^{5 / 2}$ in the $Z \rightarrow-\infty$ expression in (27), the amplitude of this periodic disturbance also decreases with increasing $\mu_{0}$ owing to the rapid decay of $\widehat{|\mathcal{A}|^{2}}(\mu)$ with increasing $\mu>1 / \sigma_{z}$. Above the wavepacket the disturbance decays rapidly to zero as $A_{0}^{2} \sigma_{x} \sigma_{z} / Z^{4}$.

\section{G. Regime diagram}

In order to gain better insight into what parameters determine whether the total induced flow will be dominated by long waves or evanescent disturbances, Figure 6 plots contours corresponding to the transition which occurs when $\mu_{0} \sigma_{z}\left(=f \sigma_{z} / c_{g z}\right) \sim 1$. Explicitly, contours 


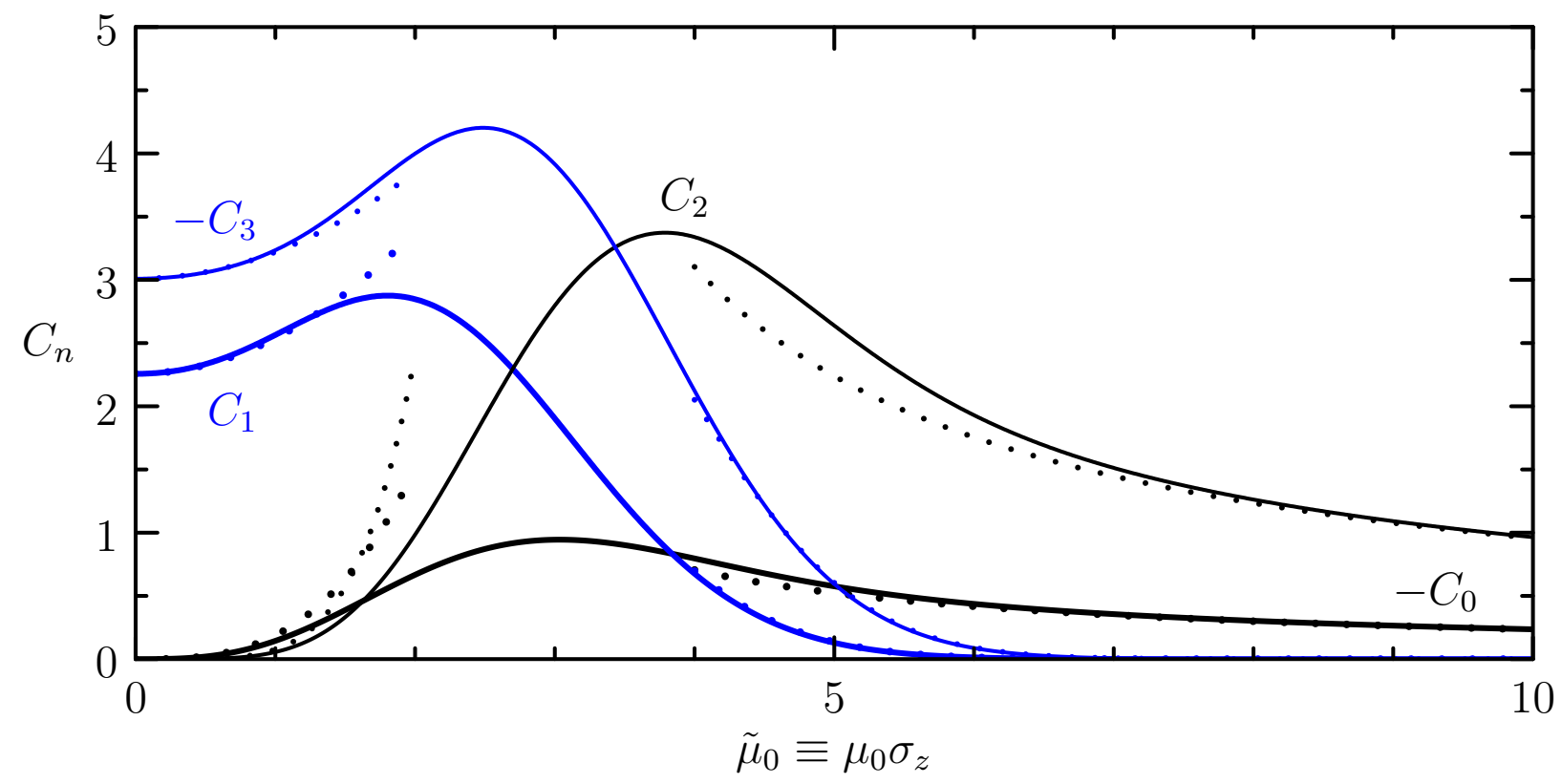

FIG. 5. Predicted horizontal flow (black curves) at $X=0$ for a) $m / k=-1$, b) -3 and c) -5 . Asymptotic approximations for $|Z| / \sigma_{z} \ll 1(25)$ and $|Z| / \sigma_{z} \gg 1(27)$ are plotted as short- and long-dashed lines, respectively.

of $k / \mu_{0}$ are plotted as they depend upon $|f| / N$ and $|m / k|$. At transition, these contour values are equal to $k \sigma_{z} / \tilde{\mu}_{0}^{\star}$.

In the examples presented above we considered a Gaussian wavepacket with $k \sigma_{z}=20$. Figure 6 shows that the case with $m=-k$ and $f=0.01 N$ has $k / \mu_{0} \simeq 35$. Being larger than $k \sigma_{z}$ means the induced flow is long-wave dominated. In contrast, the cases with $m=-3 k$ and $-5 k$ respectively have $k / \mu_{0} \simeq 9.5$ and 3.8 , and so these are dominated by evanescent disturbances. Clearly whether or not the induced flow is long-wave dominated depends upon the vertical scale of modulations of the wavepacket. If $k \sigma_{z}=5$, long waves should be dominant even in the case $m=-3 k$.

Although not part of this study, it is relevant to emphasize an important aspect of the transition from long waves to evanescent disturbances. In the former case, the horizontal flow over the wavepacket is positive on the leading flank and negative on the trailing flank. Consequently, if the waves in the packet are hydrostatic and of such large amplitude that weakly nonlinear effects become important, then the Doppler shifting of the waves by the induced flow on the trailing flank would lead to modulational instability. This is because the negative flow on the trailing flank would increase the extrinsic frequency of the waves causing their vertical group velocity to increase. Hence the amplitude envelope in the lee of the wavepacket would narrow and grow in amplitude, as has been investigated in studies of horizontally periodic (one-dimensional) wavepackets ${ }^{21-23}$ and non-rotating two-dimensional wavepackets $^{24}$. However, if the waves dominately induce an evanescent disturbance, then the flow is negative over the center of the wavepacket. Hence, hydrostatic, moderately large amplitude waves should be modulationally unstable, in contrast with the modulational 


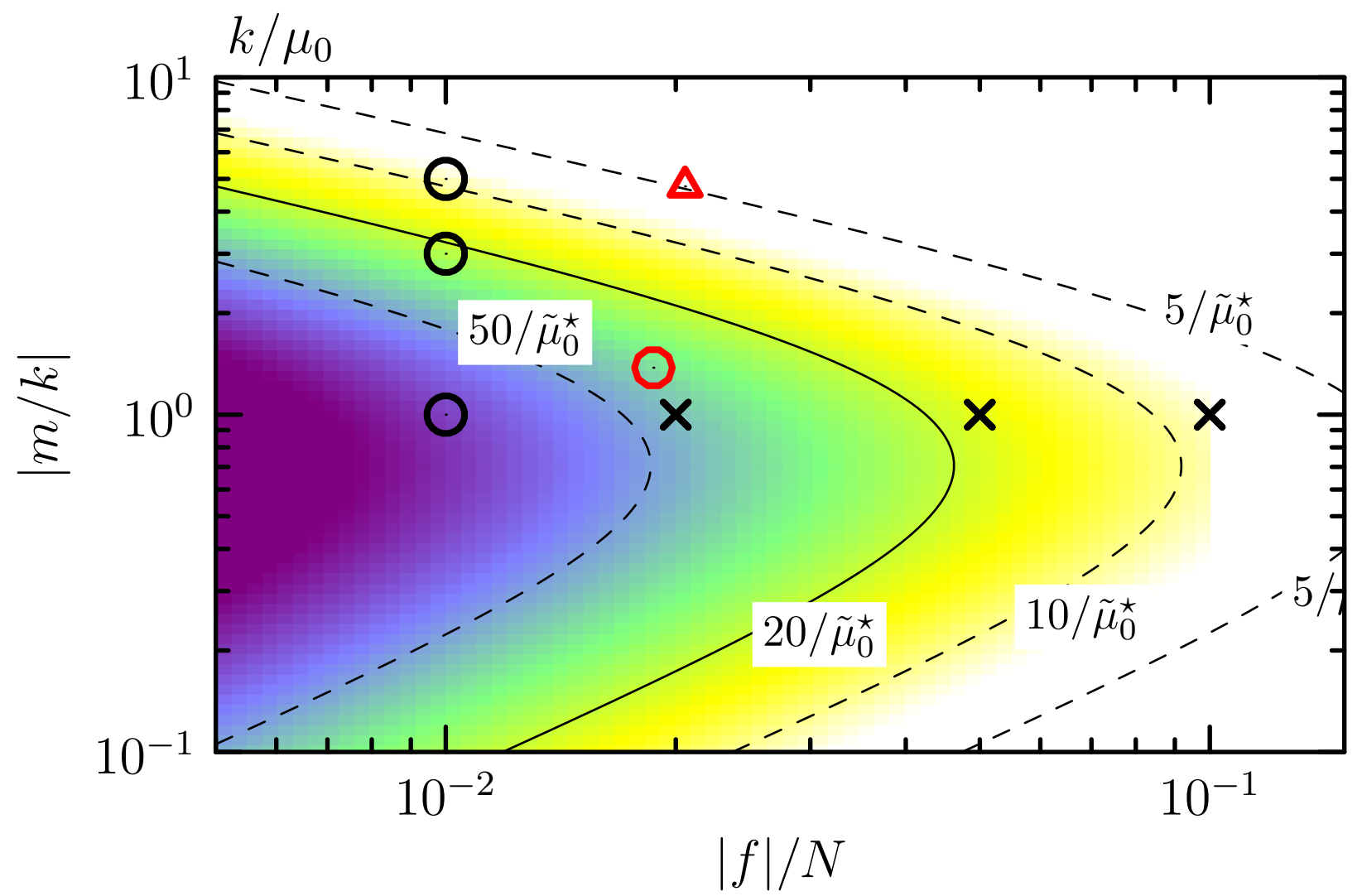

FIG. 6. Contours of $k / \mu_{0}\left(=k \sigma_{z} / \tilde{\mu}_{0}^{\star}\right.$ at transition $)$ indicating whether the induced flow is dominated by long waves or evanescent disturbances. For given $k \sigma_{z}$, parameter values to the left (right) of this contour are dominated by long waves (evanescent disturbances). A solid line is drawn for the contour with $k \sigma_{z} / \tilde{\mu}_{0}^{\star}=20 /(2.4)$, which is used for the examples presented here. The open circles indicate the parameters corresponding to the theoretical results shown in Figures $1-3$. The crosses indicate the parameters corresponding to numerical simulation results presented in $\S I I I$.

stability associated with one-dimensional and three dimensional wavepackets whose induced flow is predominately positive over the wavepacket.

The regime diagram in Fig. 6 also shows that the transition from long-wave to evanescent dominated induced flows can be obtained by fixing $|m / k|$ and increasing $|f| / N$. This is the approach taken below in performing numerical simulations.

\section{NUMERICAL SIMULATIONS}

\section{A. Description of the code and set-up}

We have run fully nonlinear simulations of the evolution of small-amplitude quasimonochromatic wavepackets, using the same code as that used to simulate two-dimensional wavepackets with no background rotation ${ }^{5}$. As basic state fields, this code works with the 
spanwise vorticity, $\zeta$, buoyancy, $b$, and spanwise velocity, $v$. In all cases reported upon here the simulations were initialized with an up- and rightward propagating wavepacket with vertical displacement field given by $\xi(x, z, t=0)=A(x, z) \cos (k x+m z)$, in which we set the vertical wavenumber to be $m=-k$. The waves were contained in a Gaussian wavepacket prescribed generally by $A(x, z)=A_{0} \exp \left[-\left(x^{2} / \sigma_{x}^{2}+z^{2} / \sigma_{z}^{2}\right) / 2\right]$, for which we set $\sigma_{x}=\sigma_{z}=20 / k$ and $A_{0} k=0.01$. With this prescription, the polarization relations in Table I were used to specify the initial values of $\zeta, b$ and $v$.

Here we explore the transition from flows dominated by long waves to those dominated by evanescent disturbances by varying $f / N$ from 0.01 to 0.1 . This is done for computational convenience: simulations with larger $|m / k|$, required more vertical grid points in order to resolve the waves, and run times were longer because the vertical group velocity was smaller. Increasing $|f| / N$ can be thought of as a study of wavepackets at mid-latitudes (where $f \sim$ $\left.10^{-4} \mathrm{~s}^{-1}\right)$ propagating in strong stratification $\left(N \sim 10^{-2} \mathrm{~s}^{-1}\right.$, as in the stratosphere and oceanic thermocline) compared to those propagating in weak stratification $\left(N \sim 10^{-3} \mathrm{~s}^{-1}\right.$, as in the troposphere and mesosphere, and in the oceanic abyss).

In some simulations, only the wavepacket was specified at the outset, in which case the Eulerian induced flow was allowed to develop naturally, and a residual disturbance was also found to develop near the original position of the wavepacket. In other simulations, the predicted Eulerian induced flow was also superimposed at the initial time. The predicted streamfunction associated with the induced flow was computed in a domain with double the vertical and horizontal extent of that used in the simulations. The corresponding fields for spanwise vorticity, spanwise velocity and buoyancy were computed in Fourier space using $\zeta=-\nabla^{2} \psi$, (19) and (20), respectively. The corresponding fields in Fourier space are $\hat{\zeta}=\left(\kappa^{2}+\mu^{2}\right) \hat{\psi}, \hat{v}=-\mu_{0} \hat{\psi}$ and $\hat{b}=\left(N^{2} / c_{g z}\right)(\kappa / \mu) \hat{\psi}$. The fields were then inverse Fourier transformed, and the portion of these fields used for the smaller simulation domain was then extracted.

After the code was initialized, the basic state fields were advanced in time through the solution of the right-hand side of the equations for spanwise vorticity,

$$
\zeta_{t}=-u \zeta_{x}-w \zeta_{z}-b_{x}+f v_{z}+\mathcal{D}_{\zeta}
$$

buoyancy,

$$
b_{t}=-u b_{x}-w b_{z}-N^{2} w+\mathcal{D}_{b},
$$

and the spanwise velocity,

$$
v_{t}=-u v_{x}-w v_{z}-f u+\mathcal{D}_{v}
$$

Here $\mathcal{D}$ denotes a Laplacian diffusion operator that acted only upon horizontal wavenumbers greater than $4 k$. For these high wavenumbers, kinematic viscosity, $\nu$, was set so the Reynolds number was $\operatorname{Re}=N /\left(k^{2} \nu\right)=10^{5}$. The diffusivity in (29) was set to equal $\nu$. In most simulations, the horizontal extent of the domain was set to be $1024 \lambda$, with $\lambda=2 \pi / k$. Vertically the domain extended from $-200 / k$ to $200 / k$. The spatial resolution was set to have 16 points spanning a wavelength of the waves in the wavepacket. The code was advanced in time using a leap frog scheme for the advective terms and forward advancing for the diffusive terms. Time was advanced in steps of $0.05 / N$ up to $N t=200$. By this time, 
the wavepacket had translated vertically at the group velocity from the origin to $z \simeq 71 / k$. Thus there was negligible overlap between the initial and final Gaussian envelope.

In order to examine the flows induced by the wavepacket, the horizontal velocity field was Fourier transformed in the horizontal, the Fourier components with wavenumbers within $5 / \sigma$ of $k$ were set to zero, and the result was then inverse Fourier transformed. The resulting field we refer to as the "wavepacket-filtered flow", and we denote its horizontal velocity by $\tilde{u}$.

\section{B. Results and comparison with theory}

To begin, we present results of numerical simulations examining a wavepacket whose induced flow is strongly influenced by rotation. Explicitly, we set $f=0.1 N$, in which case $\mu_{0} \sigma_{z} \simeq 5.7>\tilde{\mu}_{0}^{\star} \simeq 2.41$, corresponding to a predicted induced flow dominated by evanescent disturbances. We examine cases with and without the predicted Eulerian induced flow initially superimposed in turn.

\section{Simulation without the predicted Eulerian induced flow initially superimposed}

In Figure 7 we show the wavepacket-filtered horizontal flow at two different times from a simulation in which only the wavepacket is superimposed at the outset. At $N t=10$ the translating wavepacket has induced a strong positive (negative) horizontal flow along its upper (lower flank), and beams of internal waves radiate above and below the wavepacket. None of these disturbances were predicted by our steady-state theory and all are a consequence of the flow field transiently adjusting to the forcing by the localized divergence of the momentum flux over the wavepacket.

By $N t=200$, the wavepacket has propagated vertically to $k z \simeq 70$, as predicted for the waves propagating with vertical group velocity $c_{g z} \simeq 0.348 N / k$. At this time there are three qualitatively different flow features evident. At the location of the wavepacket, the flow is negative with positive flows above and below. This is the structure predicted by theory. Conversely, at the original location of the wavepacket (about $z=0$ ) the flow exhibits a flow that, about $X=0$, is positive above $Z=0$ and negative below $Z=0$. Besides these features, wave beams propagate to the left and right both upward and downward, originating from the origin. This spurious disturbance is a result of the simulation not being initialized with the predicted Eulerian induced flow.

\section{Simulations with the predicted Eulerian induced flow initially superimposed}

Figure 8 shows the wavepacket-filtered horizontal flow from simulations in which the predicted Eulerian induced flow is superimposed at the outset. In the case with $f=0.1 N$, the predicted flow (Fig. 8a, left) exhibits the vertically even symmetry in the horizontal velocity, characteristic of evanescent disturbances. Comparing the wavepacket-filtered flow at $N t=200$ (Fig. 8a, right) with that in the simulation having no induced flow initially 


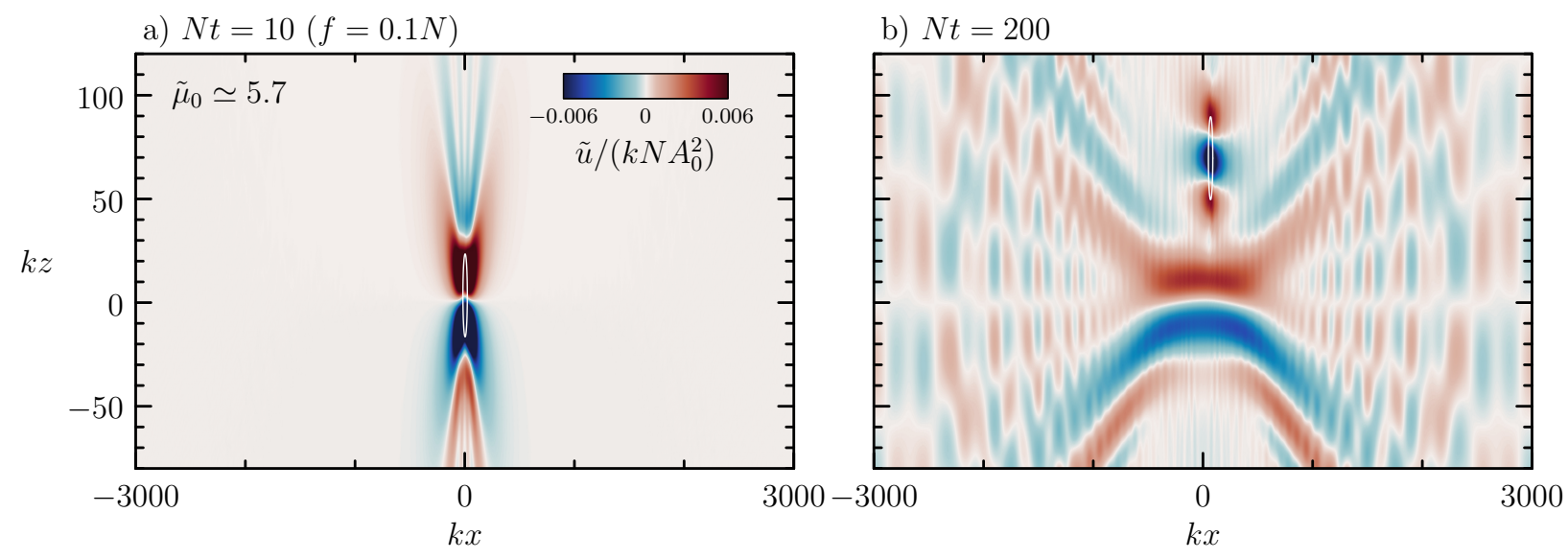

FIG. 7. Results of numerical simulation with $f=0.1 N$ and initialized only with a Gaussian wavepacket (without the predicted Eulerian induced flow initially superimposed) having $m=-k$, $A_{0}=0.01 / k$ and $\sigma_{x}=\sigma_{z}=20 / k$, showing the wavepacket-filtered horizontal flow a) at $N t=10$, and b) after evolving to time $N t=200$. In both plots, the colour scale is the same, as indicated in a), and the white circles of radii $20 / k$ indicate the location and extent of the wavepackets.

superimposed (Fig. 7b) we see that there is no significant remnant flow near the origin, and the flow around the height of the wavepacket has changed little from its initial state. Similarly, the simulations with smaller $f$, for which the predicted induced mean flow exhibits more significant long wave disturbances, the induced flow is found to translate with the wavepacket at its group velocity. These results show that numerical simulations should include the predicted Eulerian induced flow if the intent is to capture a disturbance that is steady in a frame of reference moving with the group velocity of the wavepacket.

To compare the amplitude as well as the structure of the induced flow measured in simulations with that predicted by theory, Fig. 9 plots vertical profiles of the streamwise flow at $X=0$ as predicted by theory and compares these with corresponding vertical profiles extracted at $N t=200$ from the simulations with $m=-k$ and $f=0.1 N, 0.05 N$ and $0.02 N$. In all three cases the agreement is excellent, confirming that the theory describes well the steady-state evolution of the flow induced by vertically propagating internal wavepackets.

Crucial to the development of the prediction for the induced flow is the assumption (11) that the induced flow has such broad horizontal extent compared with the extent of the wavepacket that the nonlinear forcing can be treated as a delta function in $X$. While this is a reasonable assumption if the induced flow is dominated by long waves (as in the simulation results shown Figs. 8b,c and 9b,c), it is questionable if evanescent waves dominate (as in Figs. $8 \mathrm{a}$ and $9 \mathrm{a}$ ) and the lateral extent of the evanescent disturbance is not much larger than $\sigma_{x}$. To examine this circumstance, simulations were performed with $m=-k$, as in Fig. 8, but with wavepackets having horizontal extent given by $k \sigma_{x}=60,100,150$ and 200 in the case $f=0.1 N$ and by $k \sigma_{x}=200$ in cases with $f=0.05 N$ and $f=0.02 N$. The vertical profiles of horizontal velocity through the center of the wavepacket are extracted from the simulations at $N t=200$ and compared with theory, as shown in Fig. 10. 

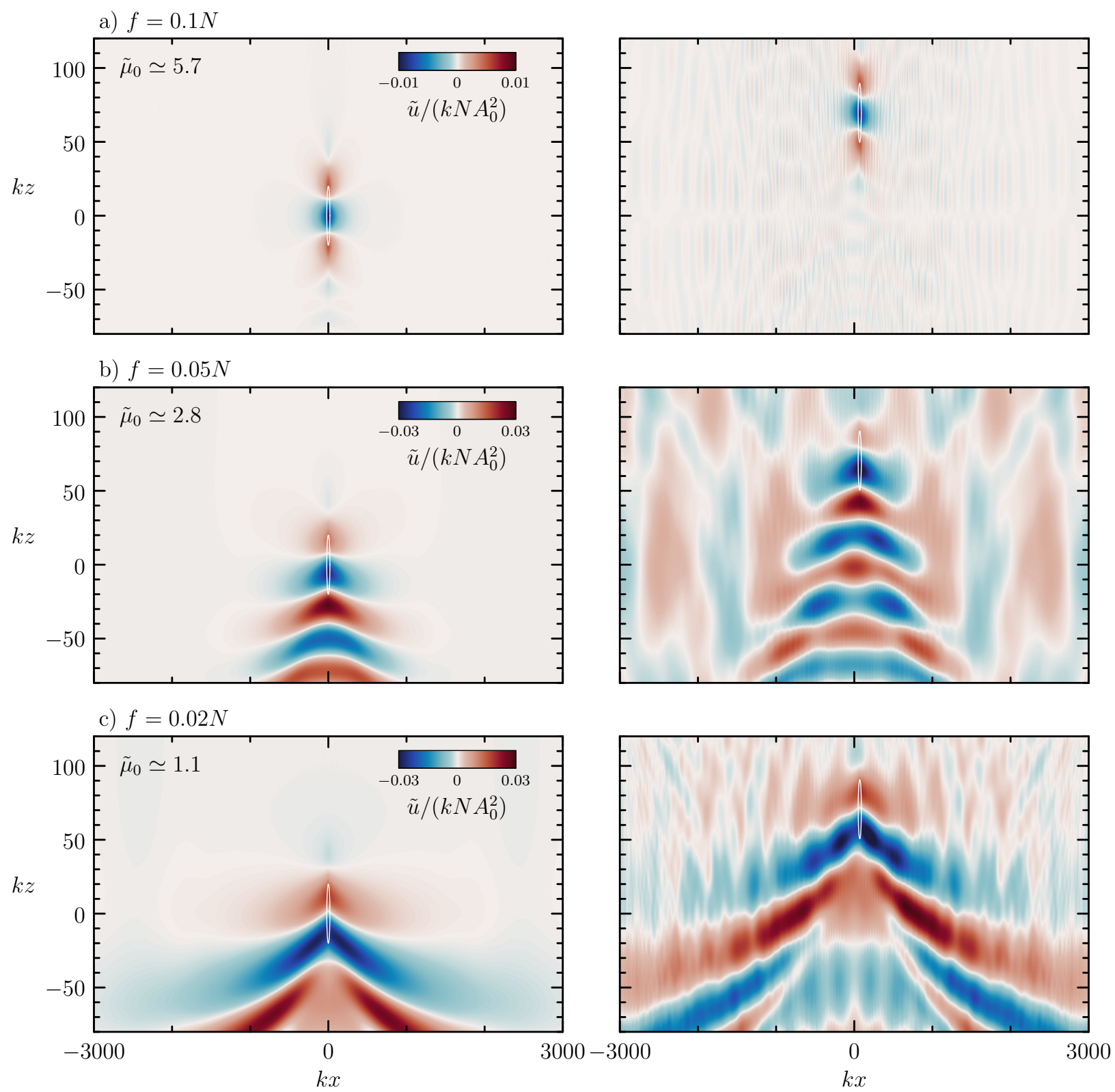

FIG. 8. Results from simulations with a) $f=0.1 N$, b) $f=0.05 N$ and c) $f=0.02 N$ showing (left) the wavepacket-filtered horizontal flow at $N t=0$ and (right) the same field at $N t=200$ with the predicted Eulerian induced flow initially superimposed. The fields on the right are plotted with the same scale as that indicated in the corresponding plot to the left, and the white circles of radii $20 / k$ indicate the location and extent of the wavepackets.

In the case with $f=0.1 N$, the predicted evanescent-dominated induced flow (Fig. 8a, left plot) becomes vanishingly small a distance $100 k^{-1}$ to the left and right of the wavepacket. Theory predicts that the structure of the flow is independent of $\sigma_{x}$ but its magnitude increases linearly with $\sigma_{x}$ (through the value of $\ell_{x}$ in (18)). This is shown by the dotted curves in Fig. 10a. However, the simulations results (solid curves) show that the magnitude 

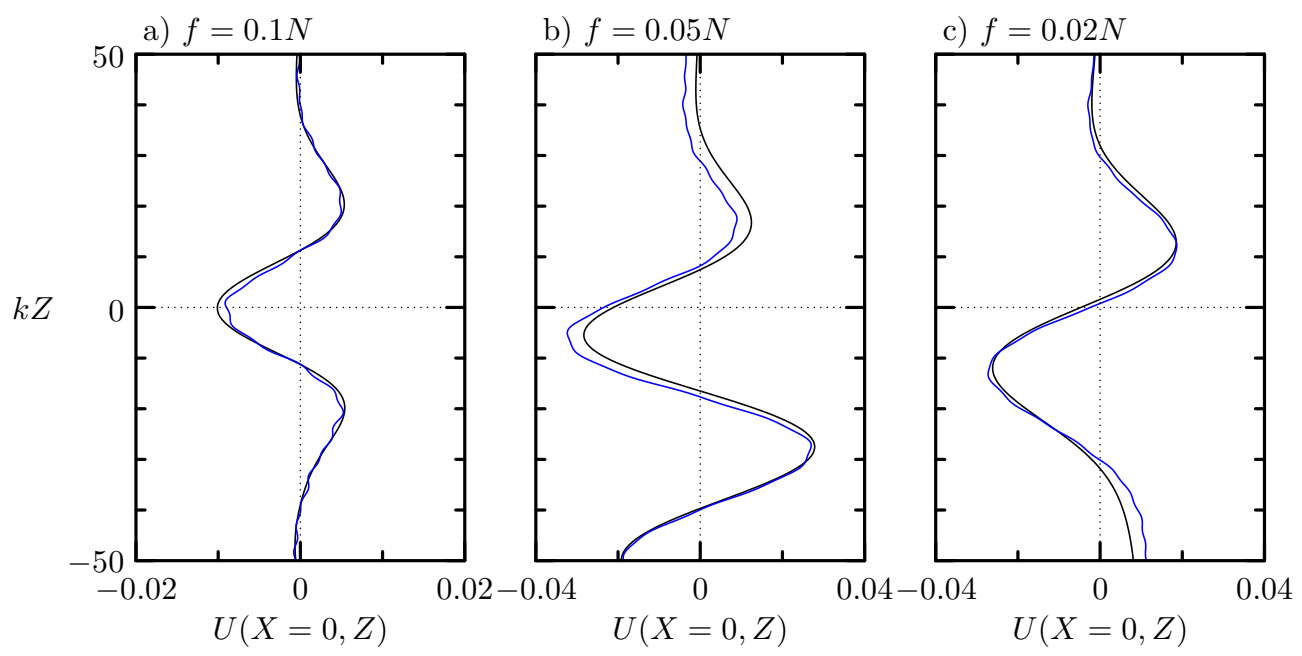

FIG. 9. Vertical profiles of the horizontal flow across the centre of the wavepacket as predicted by theory (black line) and extracted from simulations at $N t=200$ (blue lines) as shown in the right panels of Fig. 8.
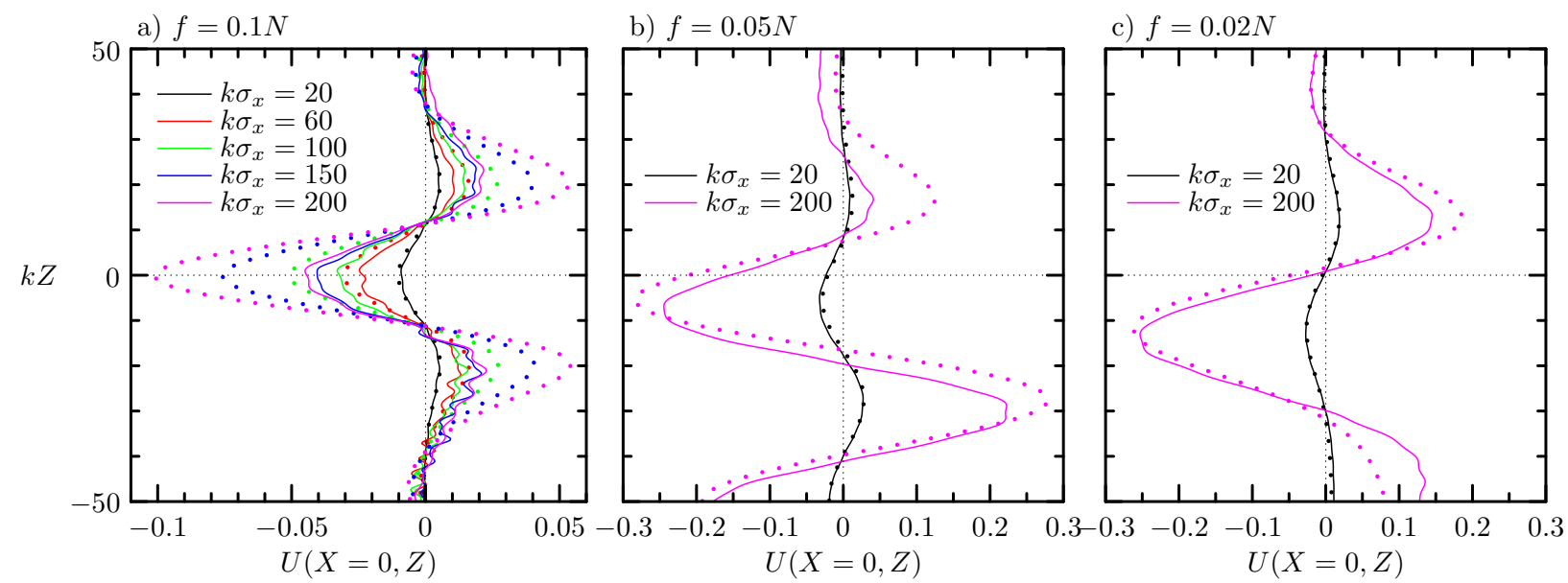

FIG. 10. As in Fig. 9, comparing vertical profiles of horizontal velocity from simulations (solid lines) with theory (dotted lines) with $m=-k$, a) $f=0.1 N$, b) $f=0.05 N$ and c) $f=0.02 N$, and with wavepacket horizontal extent $k \sigma_{x}$ as indicated by the colored lines toward the upper-left of each plot. The cases with $k \sigma_{x}=20$ are reproduced from Fig. 9 with the black dotted and solid curves corresponding, respectively, to the black and blue curves shown in Fig. 9.

of the induced flow at $X=0$ saturates as the horizontal extent of the wavepacket becomes comparable to the predicted lateral extent of the evanescent induced flow. Conversely, in the cases $f=0.05 \mathrm{~N}$ and $0.02 \mathrm{~N}$, which have a dominate long wave response with corresponding wider extent about the wavepacket, the predicted induced flow at $X=0$ corresponds well with the simulation results even with $k \sigma_{x}=200$.

It is possible to improve the theory, relaxing the assumption (11) that the response is 
so wide that the nonlinear forcing can be treated as a delta function in $X$. This would involve keeping all four terms in (9) and then solving numerically using Fourier transforms in both the horizontal and vertical directions. However, the explicit formula for the waveinduced flow (18) would seem to be robust for many atmospheric and oceanic applications, as discussed below.

\section{DISCUSSION}

Although in reality there are no truly two-dimensional wavepackets, the flow induced by modulated internal waves can behave similarly to wavepackets in the two-dimensional limit if generated with sufficiently wide lateral extent ${ }^{2}$. Explicitly, in the absence of Coriolis effects, these authors found that the induced flow from a wavepacket modulated in three dimensions takes the form of long-waves if the vertical aspect ratio, $R_{z} \equiv \sigma_{z} / \sigma_{x}$, is less than a critical value $R_{z}^{\star} \simeq 1.2\left[|k m| /\left(|\mathbf{k}|^{3} \sigma_{x}\right)\right] R_{y}^{1 / 2}$, in which $R_{y} \equiv \sigma_{y} / \sigma_{x}$ is the horizontal aspect ratio. It is natural to ask under what circumstances in the atmosphere and ocean transiently excited internal waves are sufficiently wide to satisfy this criterion.

In the ocean the most comprehensive measurements of vertically propagating waves are of those generated by tidal flow over bottom topography, such as the observations of Alford ${ }^{25}$ who recorded upward propagating near-inertial waves excited by flow over the Mendocino Ridge, or generated by storms, such the observations by Soares et $\mathrm{al}^{26}$ who recorded downward propagating waves generated by a typhoon in the tropical Indian Ocean. However, the lateral extent of the waves could not be measured and, in any case, their frequencies were close to the Coriolis frequency so that $|m / k| \gtrsim N /|f|$. Hence, only an evanescent response could occur.

A broader range of frequencies of internal waves can be excited in the atmosphere, whether by flow over topography or by convection ${ }^{27}$. (Less important mechanisms, such as imbalance caused by baroclinic instability of the jet-stream ${ }^{28}$, excite near- $f$ waves.) Thanks to satellite observations, LIDAR and measurements of OH-airglow in the upper mesosphere, as well as in situ measurements by aircraft and radiosondes, several comprehensive campaigns have recently documented the life-cycle and climatology of atmospheric internal waves ${ }^{29}$. In particular, Taylor et $\mathrm{al}^{30}$ observed over the southern island of New Zealand several northsouth oriented internal waves whose lateral extent was as large as $400 \mathrm{~km}$, containing waves with horizontal wavelengths ranging from $46-68 \mathrm{~km}$. The vertical wavelengths were such that $|m / k| \simeq 4.5-5.1$. Taking the background stratification near the top of the mesosphere to be $N \simeq 0.005$, these waves correspond to the red triangle shown in the regime diagram of Fig. 6. If the vertical extent of modulations of the wavepacket is $\sigma \simeq 20 \mathrm{~km}$, then $k \sigma_{z} / \tilde{\mu}^{\star} \simeq 2$. Hence these waves should induce a mean flow in the form of long-waves.

As another example, we consider the observations of Yue et $\mathrm{al}^{31}$ who used $\mathrm{OH}$-airglow measurements to examine circularly spreading internal waves near the mesopause. These were generated below by summer thunderstorms in north-east Colorado, USA. These waves were non-hydrostatic with horizontal wavelengths of $44 \mathrm{~km}$ near the mesopause and vertical wavelengths (inferred from the AIRS satellite) ranging between 35 and $55 \mathrm{~km}$. The characteristics of the waves are shown by the red circle in Fig. 6. The circular arcs associated 
with internal waves had largest observed diameters of around $600 \mathrm{~km}$, again suggesting a sufficiently long spanwise extent that induced flows in the form of long-waves may be possible. Indeed, assuming the waves are modulated on the scale of their wavelengths, so that $k \sigma_{x} \simeq k \sigma_{z} \simeq 6$, and taking $\sigma_{y} \simeq 300 \mathrm{~km}$, the critical vertical aspect ratio is $R_{z}^{\star} \simeq 0.2$. Thus the observed vertical extent of the waves is only moderately larger than the scale predicted for long wave resonance suggesting there may be some signature of long waves. Furthermore, because $k \sigma_{z} / \tilde{\mu}^{\star} \simeq 3$, these waves also lie in the regime such that the induced flow excites long waves.

\section{CONCLUSIONS}

We have formulated analytic solutions for the Eulerian flow induced by two-dimensional vertically propagating internal wavepackets including the effects of background rotation. The predictions were confirmed with the results of numerical simulations. Even for moderately non-hydrostatic waves, the induced flow qualitatively changes structure as determined by the value of $\mu_{0} \sigma_{z}$, in which $\mu_{0}=f / c_{g z}$ and $\sigma_{z}$ is a measure of the vertical extent of the wavepacket. For $\tilde{\mu}_{0} \equiv \mu_{0} \sigma_{z} \ll \tilde{\mu}_{0}^{\star}=2.4$, long waves dominate the induced flow which, for an up- and rightward propagating wavepacket, is positive on the upper flank and negative on the lower flank. For $\tilde{\mu}_{0} \gg \tilde{\mu}_{0}^{\star}$, evanescent disturbances dominate the induced flow. This flow is localized near the wavepacket with negative flow over the wavepacket itself. This change in flow direction across the wavepacket is expected qualitatively to change the modulational stability of moderately large amplitude waves.

Most observed vertically propagating internal wavepackets in the atmosphere and ocean are near-inertial, so their induced flow is expected to be dominated by evanescent disturbances. However, recent observations of mountain waves and of convectively-generated internal waves in the i middle atmosphere suggest that, in some circumstances, they may be non-hydrostatic and have long lateral extent as they propagate upward. In particular, because convectively-generated waves spread radially from their localized source near the top of the troposphere, the structure of their induced flow may change as they widen sufficiently to allow for long-wave resonance. Because the waves also grow in amplitude due to anelastic effects, the induced flow may have significant impact upon the evolution and consequent breaking level of the waves. This work has focused upon waves of such small amplitude that the induced flow has negligible weakly nonlinear influence upon the evolution of the waves through Doppler-shifting that can lead to enhanced amplitude growth through (modulational instability) causing the waves to overturn at altitudes lower than predicted by linear theory, or can enhance vertical dispersion (modulational stability) leading to breaking at relatively higher altitudes ${ }^{21,23,24}$. Consequently, the change in the structure of the mean flow predicted by our theory as it depends upon $|m / k|,|f| / N$ and $k \sigma_{z} / \mu_{0}^{\star}$ suggests that this should be an important consideration in predicting the ultimate level of breaking

and momentum deposition. Ongoing work is examining such weakly and fully nonlinear dynamics. 


\section{ACKNOWLEDGMENTS}

We are grateful to the reviewers for their helpful suggestions. This research was performed in part through funding from the Natural Sciences and Engineering Research Council (NSERC) of Canada and benefited from supercomputer time allocated by Compute Canada. TSvdB was supported by the Faculty of Science at the University of Alberta through its Visiting Fellowship program and by a Royal Academy of Engineering Research Fellowship.

* Email: bruce.sutherland@ualberta.ca

1 O. Bühler, Waves and Mean Flows, 2nd ed. (Cambridge University Press, Cambridge, UK, 2014) p. 341.

2 T. S. van den Bremer and B. R. Sutherland, "The wave-induced flow of internal gravity wavepackets with arbitrary aspect ratio," J. Fluid Mech. 834, 385-408 (2018), doi:10.1017/jfm.2017.745.

3 F. P. Bretherton, "On the mean motion induced by gravity waves," J. Fluid Mech. 36, 785-803 (1969).

4 A. Tabaei and T. R. Akylas, "Resonant long-short wave interactions in an unbounded rotating stratified fluid," Stud. Appl. Maths 119, 271-296 (2007).

5 T. S. van den Bremer and B. R. Sutherland, "The mean flow and long waves induced by two-dimensional internal gravity wavepackets," Phys. Fluids 26, 106601:1-23 (2014), doi:10.1063/1.4899262.

6 O. Bühler and M. E. McIntyre, "On non-dissipative wave-mean interactions in the atmosphere or oceans," J. Fluid Mech. 354, 301-343 (1998).

7 O. Bühler and M. E. McIntyre, "Remote recoil: A new wave-mean interaction effect," J. Fluid Mech. 492, 207-230 (2003).

8 J.-H. Xie and J. Vanneste, "A generalised-Lagrangian-mean model of the interactions between near-inertial waves and mean flow," J. Fluid Mech. 774, 143-169 (2015).

9 G. L. Wagner and W. R. Young, "Available potential vorticity and wave-averaged quasigeostrophic flow," J. Fluid Mech. 785, 401-424 (2015).

10 J. Thomas, O. Bühler, and K. Shafer Smith, "Wave-induced mean flows in rotating shallow water with uniform potential vorticity," J. Fluid Mech. 839, 408-429 (2018).

11 B. R. Sutherland, U. Achatz, C. P. Caulfield, and J. M. Klymak, "Recent progress in modeling imbalance in the atmosphere and ocean," Phys. Rev. Fluids 4, 010501:1-22 (2019), doi:10.1103/PhysRevFluids.4.010501.

12 J. R. Booker and F. P. Bretherton, "The critical layer for internal gravity waves in shear flow," J. Fluid Mech. 27, 513-539 (1967).

13 T. J. Dunkerton, "The role of gravity waves in the quasi-biennial oscillation," J. Geophys. Res. 102, 26053-26076 (1987).

14 N. A. McFarlane, "The effect of orographically excited gravity wave drag on the general circulation of the lower stratosphere and troposphere," J. Atmos. Sci. 44, 1775-1800 (1987). 
15 S. A. Thorpe, "Breaking internal waves and turbulent dissipation," J. Mar. Res. 68, 851-880 (2010).

16 P. Clark di Leoni and P. D. Mininni, "Absorption of waves by large-scale winds in stratified turbulence," Phys. Rev. E 91, 033015 (2015).

17 R. H. J. Grimshaw, "Wave action and wave-mean flow interaction, with application to stratified shear flows," Annu. Rev. Fluid Mech. 16, 11-44 (1984).

18 D. C. Fritts and T. J. Dunkerton, "A quasi-linear study of gravity-wave saturation and selfacceleration," J. Atmos. Sci. 41, 3272-3289 (1984).

19 J.-H. Xie and J. Vanneste, "Interaction between mountain waves and shear flow in an inertial layer," J. Fluid Mech. 816, 352-380 (2017).

20 J. Wilhelm, T.R. Akylas, G. Bölöni, J. Wei, B. Ribstein, R. Klein, and U. Achatz, "Interactions between mesoscale and submesoscale gravity waves and their efficient representation in mesoscale-resolving models," J. Atmos. Sci. 75, 2257-2280 (2018).

21 B. R. Sutherland, "Weakly nonlinear internal wavepackets," J. Fluid Mech. 569, 249-258 (2006).

22 H. V. Dosser and B. R. Sutherland, "Weakly nonlinear non-Boussinesq internal gravity wavepackets," Physica D 240, 346-356 (2011).

23 H. V. Dosser and B. R. Sutherland, "Anelastic internal wavepacket evolution and stability," J. Atmos. Sci. 68, 2844-2859 (2011).

24 A. Gervais, G. E. Swaters, T. S. van den Bremer, and B. R. Sutherland, "Evolution and stability of two-dimensional anelastic internal gravity wavepackets," J. Atmos. Sci. 75, 3703-3724 (2018), doi:10.1175/JAS-D-17-0388.1.

25 M. H. Alford, "Sustained, full-water-column observations of internal waves and mixing near Mendocino Escarpment," J. Phys. Oceanogr. 40, 2643-2660 (2010).

26 S. M. Soares, A. Natarov, and K. J. Richards, "Internal swells in the tropics: Near-inertial wave energy fluxes and dissipation during CINDY," J. Geophys. Res. 121, 3297-3324 (2016).

27 L. Hoffman, X. Xue, and M. J. Alexander, "A global view of stratospheric gravity wave hotspots located with Atmospheric Infrared Sounder observations," J. Geophys. Res. 118, 416434 (2013).

28 R. Plougonven, H. Teitelbaum, and V. Zeitlin, "Inertia gravity wave generation by the tropospheric midlatitude jet as given by the Fronts and Atlantic Storm-Track Experiment radio soundings," J. Geophys. Res. 108, 4686 (2003).

29 D. C. Fritts, R. B. Smith, M. J. Taylor, J. D. Doyle, S. D. Eckermann, A. Dörnbrack, M. Rapp, B. P. Williams, P.-D. Pautet, K. Bossert, N.R. Criddle, C. A. Reynolds, P. A. Reinecke, M. Uddstrom, M. J. Revell, R. Turner, B. Kaifler, J. S. Wagner, T. Mixa, C. G. Kruse, A. D. Nugent, C. D. Watson, S. Gisinger, S. M. Smith, R. S. Lieberman, B. Laughman, J. J. Moore, W. O. Brown, J. A. Haggerty, A. Rockwell, G. J. Stossmeister, S. F. Williams, G. Hernandez, D. J. Murphy, A. R. Klekociuk, I. M. Reid, and J. Ma, "The Deep Propagating Gravity Wave Experiment (DEEPWAVE): An airbourne and ground-based exploration of gravity wave propagation and effects from their sources throughout the lower and middle atmosphere," Bull. Amer. Meteor. Soc. 97, 425-453 (2016), doi:10.1175/BAMS-D-14-00269.1.

30 M. J. Taylor, P.-D. Pautet, D. C. Fritts, B. Kaifler, S. M. Smith, Y. Zhao, N. R. Criddle, P. McLaughlin, W. R. Pendleton Jr., M. P. McCarthy, G. Hernandez, S. D. Eckermann, J. Doyle, 
M. Rapp, B. Liley, and J. M. Russell III, "Large-amplitude mountain waves in the mesosphere observed on 21 June 2014 during DEEPWAVE: 1. Wave development, scales, momentum fluxes, and environmental sensitivity," J. Geophys. Res. 124, 10,364-10,384 (2019).

31 J. Yue, L. Hoffmann, and M. J. Alexander, "Simultaneous observations of convective gravity waves from a ground-based airglow imager and the AIRS satellite experiment," J. Geophys. Res. 118, 3178-3191 (2013). 